\title{
Long-range versus short-range correlations in the two-neutron transfer reaction ${ }^{64} \mathrm{Ni}\left({ }^{18} \mathrm{O},{ }^{16} \mathrm{O}\right){ }^{66} \mathrm{Ni}$
}

\author{
B. Paes, ${ }^{1}$ G. Santagati, ${ }^{2}$ R. Magana Vsevolodovna, ${ }^{3,4}$ F. Cappuzzello, ${ }^{2,5}$ D. Carbone, ${ }^{2}$ E. N. Cardozo,${ }^{1}$ M. Cavallaro, ${ }^{2}$ \\ H. García-Tecocoatzi, ${ }^{3}$ A. Gargano, ${ }^{6}$ J. L. Ferreira, ${ }^{1}$ S. M. Lenzi, ${ }^{7}$ R. Linares, ${ }^{1}$ E. Santopinto, ${ }^{3}$ A. Vitturi, ${ }^{8}$ and J. Lubian ${ }^{1}$ \\ ${ }^{1}$ Instituto de Física, Universidade Federal Fluminense, 24210-340, Niterói, Rio de Janeiro, Brazil \\ ${ }^{2}$ INFN, Laboratori Nazionali del Sud, I-95125, Catania, Italy \\ ${ }^{3}$ INFN, Sezione di Genova and Università di Genova, via Dodecaneso 33, I-16146 Genova, Italy \\ ${ }^{4}$ Dipartimento di Fisica dell'Università di Genova, via Dodecaneso 33, I-16164 Genova, Italy \\ ${ }^{5}$ Dipartimento di Fisica e Astronomia, Università di Catania, I-95125, Catania, Italy \\ ${ }^{6}$ Istituto Nazionale di Fisica Nucleare, Sezione di Napoli, I-80126 Napoli, Italy \\ ${ }^{7}$ INFN, Sezione di Padova, via Marzolo, 8, I-35131 Padova, Italy \\ ${ }^{8}$ Dipartimento di Fisica e Astronomia Galileo Galilei, Università di Padova, via Marzolo, 8, I-35131 Padova, Italy
}

(Received 4 August 2017; published 18 October 2017)

\begin{abstract}
Recently, various two-neutron transfer studies using the $\left({ }^{18} \mathrm{O},{ }^{16} \mathrm{O}\right)$ reaction were performed with a large success. This was achieved because of a combined use of the microscopic quantum description of the reaction mechanism and of the nuclear structure. In the present work we use this methodology to study the two-neutron transfer reaction of the ${ }^{18} \mathrm{O}+{ }^{64} \mathrm{Ni}$ system at $84 \mathrm{MeV}$ incident energy, to the ground and first $2^{+}$excited state of the residual ${ }^{66} \mathrm{Ni}$ nucleus. All the experimental data were measured by the large acceptance MAGNEX spectrometer at the Instituto Nazionale di Fisica Nucleare -Laboratori Nazionali del Sud (Italy). We have performed exact finite range cross section calculations using the coupled channel Born approximation (CCBA) and coupled reaction channel (CRC) method for the sequential and direct two-neutron transfers, respectively. Moreover, this is the first time that the formalism of the microscopic interaction boson model (IBM-2) was applied to a two-neutron transfer reaction. From our results we conclude that for two-neutron transfer to the ground state of ${ }^{66} \mathrm{Ni}$, the direct transfer is the dominant reaction mechanism, whereas for the transfer to the first excited state of ${ }^{66} \mathrm{Ni}$, the sequential process dominates. A competition between long-range and short-range correlations is discussed, in particular, how the use of two different models (Shell model and IBM's) help to disentangle long- and short-range correlations.
\end{abstract}

DOI: 10.1103/PhysRevC.96.044612

\section{INTRODUCTION}

It is well known that the atomic nucleus is a complex many-body system. The knowledge of the internal degrees of freedom is crucial to understand nuclear structure features like single-particle and collective states, clustering, pairing correlations, and other properties [1,2]. These information can be extracted from elastic, inelastic, transfer, and other nuclear reactions.

Transfer reactions have been extensively studied during the last decades. The particle(s) and cluster pickup and stripping are among the most studied reactions because of their sensitivity to the nuclear structure of the interacting partners. Two-nucleon transfer reactions have gained special attention because they can probe pairing correlations in nuclei [3-13]. In addition, they are relevant to model more complex processes such as double charge exchange reactions, which are of interest for applications in neutrinoless double beta decay researches $[14,15]$. To understand the reaction mechanism one has to elucidate if the two-neutron transfer reaction occurs in one step, under strong influence of pairing correlations, or in two steps (sequentially). In the case of one-step transfer reactions one has to identify if the ground state of the residual nucleus is fed from the ground state of the target, or if there are intermediate transitions through inelastic excitation of the projectile or target, before the transfer process occurs. These kinds of intermediate processes are very important in some cases $[8,16]$.

The $\left({ }^{18} \mathrm{O},{ }^{16} \mathrm{O}\right)$ two-neutron transfer reactions came as a promising option to substitute the $(\mathrm{t}, \mathrm{p})$ reactions [3]. Although the $(t, p)$ reactions could allow for a better experimental resolution in the measured energy spectra (as it involves reactions with lighter particles), the use of triton beams is at present strongly limited because of radiation safety. Moreover, the ${ }^{18} \mathrm{O}$ nucleus can be considered, in its ground state, as a core of ${ }^{16} \mathrm{O}$ plus two neutrons bound to it. The degree of correlation of these two "extra" neutrons can be tested in the two-neutron transfer reactions. In fact, it was shown that the diffuseness of the matter density of the ${ }^{18,17} \mathrm{O}$ nuclei is larger than the one of the core ${ }^{16} \mathrm{O}[17,18]$. This fact should increase the overlap of the projectile and target wave functions increasing the probability of these kinds of reactions.

Kahana and Baltz performed full quantum-mechanical calculations for the two-neutron transfer cross section of the $58,60,62,64 \mathrm{Ni}\left({ }^{18} \mathrm{O},{ }^{16} \mathrm{O}\right)$ reactions $[19,20]$, using the distorted wave Born approximation (DWBA). For the ${ }^{58,60} \mathrm{Ni}\left({ }^{18} \mathrm{O},{ }^{16} \mathrm{O}\right)$ reactions, they obtained experimental evidence of some significant deviations from the well-known classical bell-shaped form, when they studied the angular distribution for the transfer to the ground and to the first excited state of the residual nuclei. The angular distributions continue rising towards the most forward angles. They also studied the angular distributions for the ${ }^{62,64} \mathrm{Ni}\left({ }^{18} \mathrm{O},{ }^{16} \mathrm{O}\right)$ transfer reactions to the ground state of the residual nuclei. These angular distributions oscillate, even in the region of the maximum of the angular distribution, that resemble bell-shaped curves expected from semiclassical considerations. That anomalous behavior of the angular distributions of the ${ }^{58,60} \mathrm{Ni}\left({ }^{18} \mathrm{O},{ }^{16} \mathrm{O}\right)$ reactions and the 
normal behavior of the ${ }^{62,64} \mathrm{Ni}\left({ }^{18} \mathrm{O},{ }^{16} \mathrm{O}\right)$ reactions remains over a variety of incident energies. They claim that the reason for this forward rising of the angular distributions for these two ${ }^{58,60} \mathrm{Ni}\left({ }^{18} \mathrm{O},{ }^{16} \mathrm{O}\right)$ reactions lies in the fact that the absorption in the surface region, where the projectile and target interact, was decreased. In this way, the projectile can penetrate into the region where attractive forces dominate. A deeper understanding of these phenomena can be gained by fully quantummechanical data analyses. In particular, in Refs. $[8,9,21]$ it was found that more details about the reaction mechanism can be extracted from the data by coupled channel calculations.

To study the details of the two-neutron reaction on a target in this mass region, we have performed a high-precision measurement of the ${ }^{64} \mathrm{Ni}\left({ }^{18} \mathrm{O},{ }^{16} \mathrm{O}\right){ }^{66} \mathrm{Ni}$ reaction to the ground and to the first excited state of the residual ${ }^{66} \mathrm{Ni}$ nucleus. To describe these reactions, the structure of the relevant states of the interacting nuclei needs to be modeled as accurately as possible. The structure of ${ }^{64,66} \mathrm{Ni}$ is studied by different nuclear structure models: the shell model, the microscopic interacting boson model (IBM-2) [22], and the interacting boson fermion model (IBFM-2) [23].

Recently, in Refs. [3,16,24,25] the experimental cross sections for the two-neutron transfer reactions ${ }^{12} \mathrm{C}\left({ }^{18} \mathrm{O},{ }^{16} \mathrm{O}\right){ }^{14} \mathrm{C}$, ${ }^{16} \mathrm{O}\left({ }^{18} \mathrm{O},{ }^{16} \mathrm{O}\right){ }^{18} \mathrm{O}$, and ${ }^{13} \mathrm{C}\left({ }^{18} \mathrm{O},{ }^{16} \mathrm{O}\right){ }^{15} \mathrm{C}$ were reproduced without any free parameters. In several previous works calculations for the two-neutron transfer reaction $\left({ }^{18} \mathrm{O},{ }^{16} \mathrm{O}\right)$ on various target nuclei were performed. In some of them the direct (one-step) process was found to be the dominant transfer mechanism (see, for example, Refs. [3,6]). In other works, the two-step process (sequential) was found to be the more important one (see, for example, Ref. [12]).

In the present work we measure and perform calculations for the two-neutron transfer reaction ${ }^{64} \mathrm{Ni}\left({ }^{18} \mathrm{O},{ }^{16} \mathrm{O}\right){ }^{66} \mathrm{Ni}$ at $84 \mathrm{MeV}$, considering one- and two-step processes to check which type of mechanism is more important and consequently the effect of pairing correlation on this reaction mechanism. The effect of the inelastic excitation of the projectile and the target on the transfer reaction is also taken into account.

It was recently shown the relevance of the $1 g_{9 / 2}$ orbital for the determination of the structure properties of the even-even Nickel isotopes [26]. In this work, this orbital was included in the model space. The influence of the choice of different structure models, that allows one to derive the spectroscopic amplitudes, on the two-neutron transfer reaction cross sections is studied in detail.

The paper is organized as follows. In Sec. II the experimental details are given. Section III is devoted to the analysis of the experimental data using different theoretical approaches for the cross section calculations. Finally, in Sec. IV summary and conclusions are reported.

\section{EXPERIMENTAL DETAILS}

A beam of ${ }^{18} \mathrm{O}^{6+}$, delivered by the Tandem Van der Graaff accelerator of the INFN-LNS laboratory in Catania, bombarded a $113 \mu \mathrm{g} / \mathrm{cm}^{2}$ self-supporting ${ }^{64} \mathrm{Ni}$ target at $84 \mathrm{MeV}$ incident energy. A system of collimators was used to limit the beam spot size and the angular divergence to $1.2 \mathrm{~mm} \times$ $0.8 \mathrm{mrad}$ in the horizontal direction and $2.3 \mathrm{~mm} \times 3 \mathrm{mrad}$

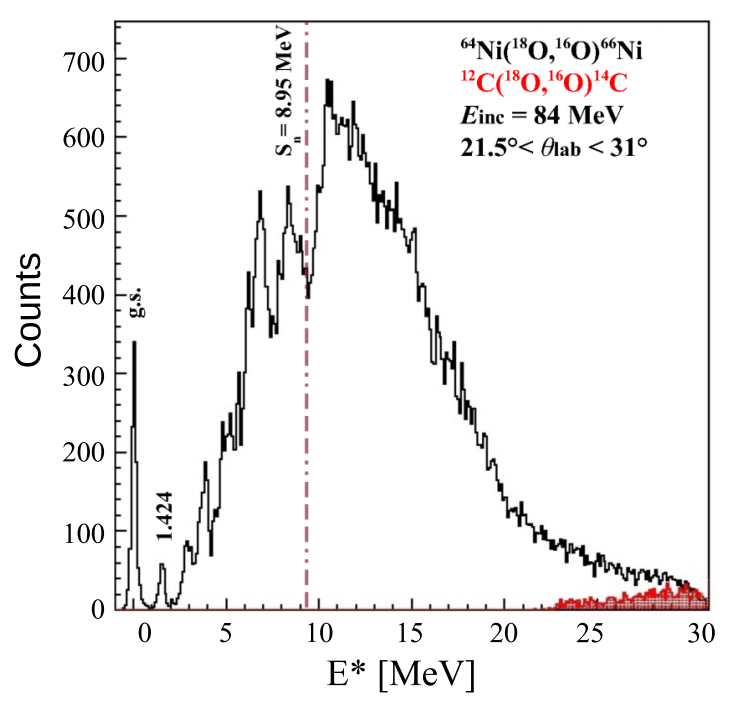

FIG. 1. Excitation energy spectrum of ${ }^{66} \mathrm{Ni}$ obtained from the ${ }^{18} \mathrm{O}+{ }^{64} \mathrm{Ni}$ reaction at $84 \mathrm{MeV}$ and $21.5^{\circ}<\theta_{\text {lab }}<31^{\circ}$. The red-hatched area corresponds with the background that comes from ${ }^{12} \mathrm{C}$ impurities in the target. Line corresponding to the one-neutron separation energy $\left(S_{n}\right)$ is also indicated.

in the vertical one. In addition, supplementary runs were performed bombarding a $49 \mu \mathrm{g} / \mathrm{cm}^{2}$ self-supporting ${ }^{12} \mathrm{C}$ target with the same beam at the same incident energy to evaluate the background in the spectra from carbon impurities in the target.

The outgoing ejectiles, produced in the different reactions were momentum analyzed by the MAGNEX spectrometer $[25,27]$ (solid angle $50 \mathrm{msr}$ ) and detected by the focal plane detector FPD [28]. The optical axis was centered at $\theta_{\text {lab }}=6^{\circ}$, $12^{\circ}, 18^{\circ}, 24^{\circ}$ central laboratory angles. Considering the large acceptance of the spectrometer, an overall angular interval of $3^{\circ}<\theta_{\mathrm{lab}}<31^{\circ}$ in the laboratory system was spanned. In addition, the large acceptance provides a fairly wide angular overlap between two consecutive settings. The magnetic field was set to transport the ${ }^{16} \mathrm{O}$ ejectiles, corresponding to the population of the ground state of the ${ }^{66} \mathrm{Ni}$ in the ${ }^{64} \mathrm{Ni}\left({ }^{18} \mathrm{O},{ }^{16} \mathrm{O}\right){ }^{66} \mathrm{Ni}$ reaction, $7 \%$ above the reference momentum, thus exploring the excited states of the ${ }^{66} \mathrm{Ni}$ up to about $30 \mathrm{MeV}$ excitation energy (thanks to the large momentum acceptance $p_{\max } / p_{\min } \sim 1.3$ of the spectrometer).

The FPD measures all relevant parameters (positions, angles, energy loss, and residual energy) for particle identification [29] and trajectory reconstruction [30]. Details of the data reduction technique are described in Refs. [31,32].

Figure 1 shows a typical energy spectrum obtained applying the ray-reconstruction technique on the experimental data detected for $21.5^{\circ}<\theta_{\text {lab }}<31^{\circ}$ in the laboratory frame. The energy axis is referred to the ${ }^{66} \mathrm{Ni}$ excitation energy $E^{*}=$ $Q_{0}-Q$, where $Q_{0}$ is the $Q$ value $(2.862 \mathrm{MeV})$ for the ground state to ground state transition. The red histogram represents the energy spectrum of ${ }^{14} \mathrm{C}$ expressed as a function of the excitation energy of the ${ }^{66} \mathrm{Ni}$. It is necessary to subtract this contribution to obtain the energy spectrum of ${ }^{66} \mathrm{Ni}$, that is the black histogram shown in Fig. 1. 
In this spectrum the ground state and the first excited state $(E$ $=1.424 \mathrm{MeV}, J^{\pi}=2^{+}$) are clearly separated. The identification of the many peaks was confirmed by comparing the energy spectrum with the spectrum of the ${ }^{64} \mathrm{Ni}(\mathrm{t}, \mathrm{p}){ }^{66} \mathrm{Ne}$ reaction [33]. The energy resolution obtained in this experiment, estimated from the Gaussian fit of the ground-state peak (FWHM), is about $220 \mathrm{keV}$, principally limited by the energy straggling of the ${ }^{16} \mathrm{O}$ in the different crossed materials ( ${ }^{64} \mathrm{Ni}$ target, FPD Mylar window entrance, etc).

Several sharp peaks are observed, indicating a relevant population of bound and resonant states. The particular shape of this spectrum can be explained considering the high-level density of ${ }^{66} \mathrm{Ni}$ [34] and the kinematic matching conditions which gives a maximum cross section at $E_{\mathrm{opt}}^{*}=12.2 \mathrm{MeV}$ and $L_{\text {opt }}=5 \hbar$ [35]. The continuum shape observed at excitation energies higher than the neutron separation energy $\left(S_{n}=\right.$ $8.95 \mathrm{MeV}$ ) is also from the three-body kinematics connected to the one-neutron emission. The large bump in this spectrum may be associated with the competition between the twoneutron transfer mechanism and dissipative phenomena [36].

The obtained angular distributions for the transfer to the ground state and to the first excited state of ${ }^{66} \mathrm{Ni}$ are shown in Fig. 3. The angular resolution is $0.3^{\circ}$. A scale error in the cross section of $10 \%$, coming from uncertainties in the target thickness and beam integration by the Faraday cup, is common to all the angular distribution points and it is not included in the error bars. These correspond to other sources of uncertainty, such as the solid angle determination, the statistical error, and the background subtraction.

In Fig. 3 one can see that the angular distribution of the elastic transfer has a bell shape at near the grazing angle, as predicted by semiclassical results. Nevertheless, at forward angles the cross section oscillates and the mean value stops decreasing. This indicates that some transparency (not very strong absorption) is present and the attraction of the nuclear field is felt in the reactions also with the ${ }^{64} \mathrm{Ni}$. The forward angle oscillations were not observed in the experimental data of Ref. [20] because they were not able to measure the forward angles and because of the limited angular distribution.

\section{CRC AND DWBA CALCULATIONS. RESULTS, AND DISCUSSIONS}

We have performed cross section calculations using the exact finite range $\mathrm{CCBA}$ and $\mathrm{CRC}$ method. All the calculations were performed using the FRESCO [37] code. The São Paulo double folding potential (SPP) [38] was used as the optical potential for the real and imaginary parts. In the entrance partition a strength factor of 0.6 was used, for the imaginary part, according to Ref. [39]. This factor accounts for the missing couplings to dissipative processes, as well as, for the coupling to continuum states, which are not explicitly considered in the calculations. In the outgoing and intermediate partitions the strength factor for the imaginary part of the optical potential was set equal to 0.78 , because no couplings were included. This 0.78 coefficient was proved to be suitable for describing the elastic scattering cross section for many systems in a wide mass and energy intervals [40].
To generate single-particle and cluster wave functions Woods-Saxon form factors were used. The depth of these potentials was adjusted to fit the experimental separation energies for one and two neutrons. The reduced radii and diffuseness were set equal to $1.26 \mathrm{fm}$ and $0.7 \mathrm{fm}$ for binding the two neutrons to the core ${ }^{16} \mathrm{O}$ and $1.3 \mathrm{fm}$ and $0.7 \mathrm{fm}$ for binding to the ${ }^{64} \mathrm{Ni}$ core. For binding one neutron to the ${ }^{17} \mathrm{O}$ core the reduced radii and diffuseness were set equal to $1.26 \mathrm{fm}$ and $0.7 \mathrm{fm}$ and for binding it to the ${ }^{65} \mathrm{Ni}$ core they were set equal to $1.3 \mathrm{fm}$ and $0.7 \mathrm{fm}$, respectively. Similar reduced radius and diffuseness parameters were recently used to describe the two-neutron transfer reaction ${ }^{12} \mathrm{C}\left({ }^{18} \mathrm{O},{ }^{16} \mathrm{O}\right){ }^{14} \mathrm{C}$ [3], ${ }^{16} \mathrm{O}\left({ }^{18} \mathrm{O},{ }^{16} \mathrm{O}\right){ }^{18} \mathrm{O}[16]$, and ${ }^{13} \mathrm{C}\left({ }^{18} \mathrm{O},{ }^{16} \mathrm{O}\right){ }^{15} \mathrm{C}$ [41].

The deformation parameters for the quadrupole collective excitations of the projectile and the target in the entrance partition were taken from [42]. The prior representation, nonorthogonality corrections, and full complex remnant approximation were adopted for the calculations of the reaction kernels in the coupled channel equations.

Three different models were used for the calculation of the two-neutron transfer reaction. The first two models assume that the transfer reaction of the two neutrons occurs simultaneously or in a direct way. The first is the extreme cluster model, where the internal structure of the "di-neutron" cluster is completely ignored and the two neutrons are coupled antiparallel to the total spin zero in the $1 s$ state. The spectroscopic amplitudes for both target and projectile overlaps are usually set equal to 1.0 in this model. In a recent work it was shown [41] that a microscopic cluster calculation can be performed if the two-neutron spectroscopic amplitudes from extended shell model calculations are used. A unitary transformation allows one to derive the spectroscopic amplitudes of the cluster model, and the parallel and antiparallel configuration of the two neutrons inside the cluster can then be considered. In the present work we will use only the extreme cluster model because it is the simplest approach and already gives a qualitative insight into the twoneutron pairing correlation in the considered states of ${ }^{66} \mathrm{Ni}$.

The second model is the so-called independent coordinate model, where the relative movement between the two particles is taken into account, and all the quantum numbers of the individual neutrons are considered during the transfer process. The description of these two mentioned approaches can be found in Refs. [1] and [37], respectively.

The third model considers that the transfer of the two neutrons occurs in two steps, passing through an intermediate partition, known as a sequential or two-step mechanism.

For the direct two-neutron transfer reaction (or one-step mechanism), that is, for the extreme cluster and the independent coordinate models, CRC calculations were performed. For the sequential transfer (or two-step mechanism) two-step distorted wave Born approximation (DWBA) calculations were performed in post prior representation. We mention that for the sequential transfer what we perform in fact is a two-step CCBA calculation. The reason is that in the entrance partition the inelastic excitations of the ${ }^{18} \mathrm{O}$ projectile and ${ }^{64} \mathrm{Ni}$ target are considered to high orders. To emphasize that the sequential transitions between the partitions is calculated in first-order DWBA we will call this process two-step DWBA in the present work. 
(a)
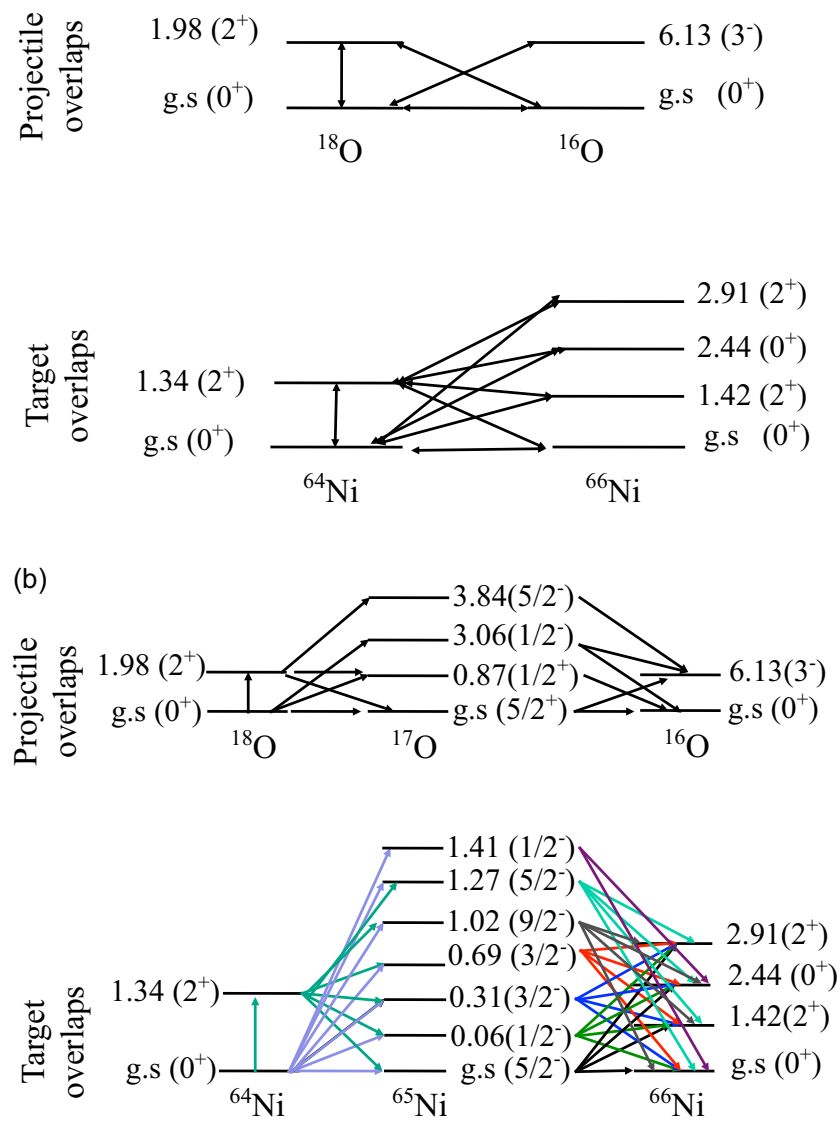

FIG. 2. Coupling scheme of the projectile and target overlaps used in the direct (a) and sequential (b) two-neutron transfer reaction calculations.

The coupling scheme of projectile and target overlaps for direct and sequential two-neutron transfer reactions adopted in the calculation are sketched in Figs. 2(a) and 2(b), respectively.

\section{A. Cluster model results}

In the extreme cluster model calculations the $f p$ shell was considered. For this reason, independently of the orbital that each neutron populates, it carries three quanta of excitation. The parameters relevant for the definition of the cluster wave function are the principal quantum number $N$ and the orbital angular momentum $L$ of the cluster relative to the core. These parameters are obtained from the conservation of the total number of quanta in the transformation of the wave function of two independent neutrons in orbits $\left(n_{i}, l_{i}\right)(i=1,2)$ into a cluster with the internal state described by the quantum numbers $(n, l)$ [43]: $2\left(n_{1}-1\right)+l_{1}+2\left(n_{2}-1\right)+l_{2}=2(N-1)+L+2(n-1)$ $+l$. As mentioned above, in the extreme cluster model, the two neutrons are assumed to be in the $1 s$ state, corresponding to the quantum numbers $n=1$ and $l=0$. The value 1.0 for the spectroscopic amplitudes is assumed and the component $S=1$, where the two neutrons are coupled parallel, is neglected. In the case that this simplification is not valid, the cross section for the
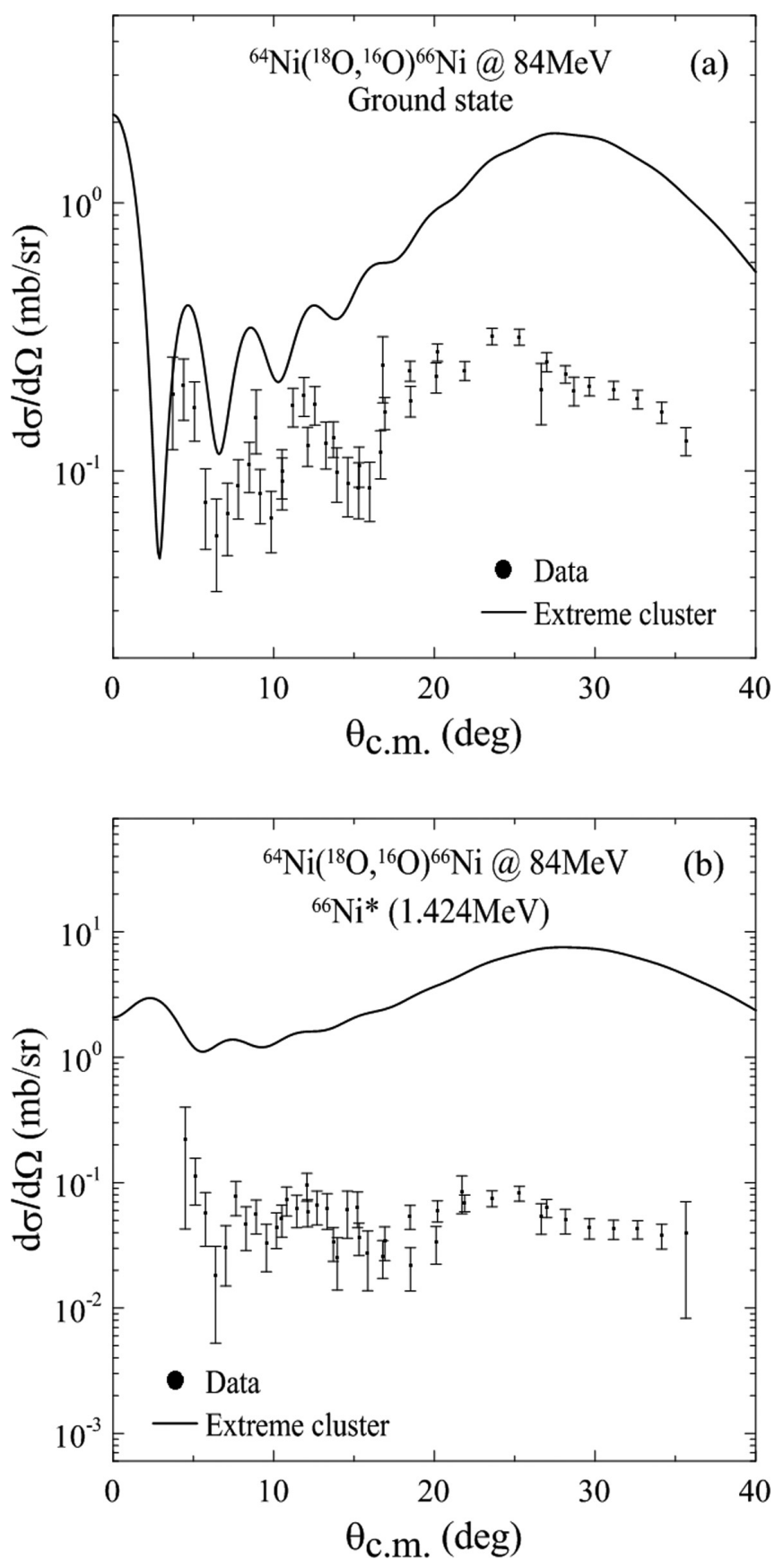

FIG. 3. Experimental angular distributions for the transitions to the ground (a) and first excited state (b) at $1.424 \mathrm{MeV}$ in ${ }^{66} \mathrm{Ni}$. The curve corresponds to the extreme cluster model results.

specific reaction might be overestimated. In other words, this extreme cluster model should give the upper limit in the case where pair correlation between the two neutrons is relevant.

In both two-neutron transfer reactions, to the ground and to the first excited state of ${ }^{66} \mathrm{Ni}$, the model overestimates the experimental data [see Figs. 3(a) and 3(b)].

\section{B. Shell-model calculations}

To perform microscopic independent coordinates and sequential calculations it is necessary to determine the 
spectroscopic amplitude for each target and projectile overlap. Two possibilities have been explored for this medium mass system, the shell model (SM) and the IBM method. In this section we will describe the former. The spectroscopic amplitudes were calculated using the NUSHELLX code [44], by performing extensive shell-model calculations. In the diagonalization of the shell-model Hamiltonian, some approximations are necessary. One usual approximation is the truncation of the valence space over some inert core. Taking this into account, the spectroscopic amplitudes were derived using the model space bjuff and the effective phenomenological interaction of Ref. [26], derived from CD-Bonn $N N$ potential renormalized by the so-called $\mathrm{V}_{\text {low }-k}$ approach $[45,46]$. This interaction was developed exclusively for Nickel isotopes with mass near the one of ${ }^{64} \mathrm{Ni}$.

In the bjuff model space the valence particles can populate the $1 f_{7 / 2}$ and $2 p_{3 / 2}$ orbitals for protons and the $2 p_{3 / 2}, 1 f_{5 / 2}$, $2 \mathrm{p}_{1 / 2}$, and $1 \mathrm{~g}_{9 / 2}$ orbitals for neutrons. The spectroscopic amplitudes for two-neutron transfer cross section calculations using the ${ }^{48} \mathrm{Ca}$ as core, with the model space bjuff and the effective phenomenological interaction of Ref. [26] are listed in Table I for direct transfer, and Tables II and III for the two-step process. This model space allowed one to describe the experimental energy spectra of ${ }^{64} \mathrm{Ni},{ }^{65} \mathrm{Ni}$, and ${ }^{66} \mathrm{Ni}$ isotopes with good agreement with difference lower than $200 \mathrm{keV}$ up to the excitation energy of $1.34 \mathrm{MeV}$ for ${ }^{64} \mathrm{Ni}, 1.41 \mathrm{MeV}$ for ${ }^{65} \mathrm{Ni}$, and $2.91 \mathrm{MeV}$ for ${ }^{66} \mathrm{Ni}$ isotopes, for both negative- and positive-parity states.

The spectroscopic amplitudes for the projectile overlaps were taken from Ref. [41], where it was shown that the zbm interaction [47] is suitable for describing both energy spectra of carbon and oxygen isotopes as well as neutron transfer reactions. The obtained results are compared with the experimental data in Fig. 4.

\section{The microscopic IBM-2 (one-step) calculation}

Here we describe the construction of the nuclear wave functions of the even-even nuclei ${ }^{66,64} \mathrm{Ni}$ within the microscopic IBM-2 by using the parameters given in Table IV. The obtained spectra are shown in Fig. 5, and one can see that the low-lying states of these nuclei are in agreement with the experimental data.

We computed for the first time the spectroscopic amplitudes of the two-neutron transfer reactions, using the microscopic IBM-2 for the ${ }^{64} \mathrm{Ni}$ and ${ }^{66} \mathrm{Ni}$ overlaps. The microscopic IBM-2 is a way to calculate realistic matrix elements for medium and heavy nuclei, that was applied recently in neutrinoless double- $\beta$ decay [48].

To derive the spectroscopic amplitudes of the two-nucleon transfer reactions a mapping of the two-body matrix elements for the nonconservative operator in the generalized seniority scheme [49] is performed.

In the microscopic IBM [50], the shell-model $S D$ pair states are mapped onto $s d$ boson states with $J^{\pi}=0^{+}$and $J^{\pi}=2^{+}$,

$$
\begin{aligned}
& S^{\dagger} \rightarrow s^{\dagger}, \\
& D^{\dagger} \rightarrow d^{\dagger} .
\end{aligned}
$$

TABLE I. Spectroscopic amplitudes used in the CRC calculations for two-neutron transfer using the model space bjuff with the effective phenomenological interaction of Ref. [26] and ${ }^{48} \mathrm{Ca}$ as an inert core. $j_{1} j_{2}$ are the spins of the neutron orbitals for two-neutron transfer, and

\begin{tabular}{|c|c|c|c|c|}
\hline Initial state & $j_{1} j_{2}$ & $J_{12}$ & Final state & Spect. ampl. \\
\hline${ }^{64} \mathrm{Ni}_{\text {g.s. }}\left(0^{+}\right)$ & $\begin{array}{l}\left(1 f_{5 / 2}\right)^{2} \\
\left(2 p_{3 / 2}\right)^{2} \\
\left(2 p_{1 / 2}\right)^{2} \\
\left(1 g_{9 / 2}\right)^{2}\end{array}$ & 0 & ${ }^{66} \mathrm{Ni}_{g . s .}\left(0_{1}^{+}\right)$ & $\begin{array}{r}0.765 \\
0.368 \\
0.301 \\
-0.278\end{array}$ \\
\hline${ }^{64} \mathrm{Ni}_{\text {g.s. }}\left(0^{+}\right)$ & $\begin{array}{c}\left(1 f_{5 / 2}\right)^{2} \\
\left(1 f_{5 / 2} 2 p_{3 / 2}\right) \\
\left(1 f_{5 / 2} 2 p_{1 / 2}\right) \\
\left(2 p_{3 / 2}\right)^{2} \\
\left(2 p_{3 / 2} 2 p_{1 / 2}\right) \\
\left(1 g_{9 / 2}\right)^{2}\end{array}$ & 2 & ${ }^{66} \mathrm{Ni}_{1.424}\left(2_{1}^{+}\right)$ & $\begin{array}{r}0.052 \\
-0.071 \\
0.200 \\
0.001 \\
-0.029 \\
-0.071\end{array}$ \\
\hline${ }^{64} \mathrm{Ni}_{\text {g.s. }}\left(0^{+}\right)$ & $\begin{array}{l}\left(1 f_{5 / 2}\right)^{2} \\
\left(2 p_{3 / 2}\right)^{2} \\
\left(2 p_{1 / 2}\right)^{2} \\
\left(1 g_{9 / 2}\right)^{2}\end{array}$ & 0 & ${ }^{66} \mathrm{Ni}_{2.445}\left(0_{2}^{+}\right)$ & $\begin{array}{c}-0.031 \\
-0.117 \\
-0.372 \\
0.360\end{array}$ \\
\hline${ }^{64} \mathrm{Ni}_{\text {g.s. }}\left(0^{+}\right)$ & $\begin{array}{c}\left(1 f_{5 / 2}\right)^{2} \\
\left(1 f_{5 / 2} 2 p_{3 / 2}\right) \\
\left(1 f_{5 / 2} 2 p_{1 / 2}\right) \\
\left(2 p_{3 / 2}\right)^{2} \\
\left(2 p_{3 / 2} 2 p_{1 / 2}\right) \\
\left(1 g_{9 / 2}\right)^{2}\end{array}$ & 2 & ${ }^{66} \mathrm{Ni}_{2.916}\left(2_{2}^{+}\right)$ & $\begin{array}{r}-0.106 \\
0.042 \\
-0.012 \\
0.007 \\
0.055 \\
-0.433\end{array}$ \\
\hline${ }^{64} \mathrm{Ni}_{1.345}\left(2^{+}\right)$ & $\begin{array}{c}\left(1 f_{5 / 2}\right)^{2} \\
\left(1 f_{5 / 2} 2 p_{3 / 2}\right) \\
\left(1 f_{5 / 2} 2 p_{1 / 2}\right) \\
\left(2 p_{3 / 2}\right)^{2} \\
\left(2 p_{3 / 2} 2 p_{1 / 2}\right) \\
\left(1 g_{9 / 2}\right)^{2}\end{array}$ & 2 & ${ }^{66} \mathrm{Ni}_{\text {g.s. }}\left(0_{1}^{+}\right)$ & $\begin{array}{r}-0.551 \\
-0.420 \\
-0.402 \\
-0.230 \\
0.303 \\
0.010\end{array}$ \\
\hline${ }^{64} \mathrm{~N}_{1.345}\left(2^{+}\right)$ & $\begin{array}{l}\left(1 f_{5 / 2}\right)^{2} \\
\left(2 p_{3 / 2}\right)^{2} \\
\left(2 p_{1 / 2}\right)^{2} \\
\left(1 g_{9 / 2}\right)^{2}\end{array}$ & 0 & ${ }^{66} \mathrm{Ni}_{1.424}\left(2_{1}^{+}\right)$ & $\begin{array}{r}0.590 \\
0.269 \\
0.115 \\
-0.213\end{array}$ \\
\hline${ }^{64} \mathrm{Ni}_{1.345}\left(2^{+}\right)$ & $\begin{array}{c}\left(1 f_{5 / 2}\right)^{2} \\
\left(1 f_{5 / 2} 2 p_{3 / 2}\right) \\
\left(1 f_{5 / 2} 2 p_{1 / 2}\right) \\
\left(2 p_{3 / 2}\right)^{2} \\
\left(2 p_{3 / 2} 2 p_{1 / 2}\right) \\
\left(1 g_{9 / 2}\right)^{2}\end{array}$ & 2 & ${ }^{66} \mathrm{Ni}_{2.445}\left(0_{2}^{+}\right)$ & $\begin{array}{r}-0.020 \\
0.001 \\
0.427 \\
0.099 \\
-0.362 \\
0.263\end{array}$ \\
\hline${ }^{64} \mathrm{~N}_{1.345}\left(2^{+}\right)$ & $\begin{array}{l}\left(1 f_{5 / 2}\right)^{2} \\
\left(2 p_{3 / 2}\right)^{2} \\
\left(2 p_{1 / 2}\right)^{2} \\
\left(1 g_{9 / 2}\right)^{2}\end{array}$ & 0 & ${ }^{66} \mathrm{Ni}_{2.916}\left(2_{2}^{+}\right)$ & $\begin{array}{r}0.084 \\
0.084 \\
0.057 \\
-0.476\end{array}$ \\
\hline${ }^{64} \mathrm{Ni}_{1.345}\left(2^{+}\right)$ & $\begin{array}{c}\left(1 f_{5 / 2}\right)^{2} \\
\left(1 f_{5 / 2} 2 p_{3 / 2}\right) \\
\left(2 p_{3 / 2}\right)^{2} \\
\left(2 p_{3 / 2}\right)^{2} \\
\left(2 p_{3 / 2} 2 p_{1 / 2}\right) \\
\left(1 g_{9 / 2}\right)^{2}\end{array}$ & 2 & ${ }^{66} \mathrm{Ni}_{1.424}\left(2_{1}^{+}\right)$ & $\begin{array}{r}-0.027 \\
-0.084 \\
-0.211 \\
-0.001 \\
-0.019 \\
0.054\end{array}$ \\
\hline
\end{tabular}
$J_{12}$ is the angular momentum of the two-neutron system.

Fermionic operators are similarly mapped into bosonic operators using the Otsuka, Arima, and Iachello (OAI) method [51]. In this method, one is assured that the matrix elements between fermionic states in the collective 
TABLE II. Spectroscopic amplitudes used in the DWBA calculations for one-neutron transfer using the model space, interaction, and core as in Table I, where $j$ is the spin of the neutron orbitals.

\begin{tabular}{|c|c|c|c|}
\hline Initial state & $j$ & Final state & Spect. Ampl. \\
\hline${ }^{64} \mathrm{Ni}_{\text {g.s }}\left(0^{+}\right)$ & $\left(1 f_{5 / 2}\right)$ & ${ }^{65} \mathrm{Ni}_{\text {g.s }}\left(5 / 2_{1}^{-}\right)$ & 0.586 \\
\hline${ }^{64} \mathrm{Ni}_{g . s}\left(0^{+}\right)$ & $\left(2 p_{1 / 2}\right)$ & ${ }^{65} \mathrm{Ni}_{0.063}\left(1 / 2_{1}^{-}\right)$ & 0.474 \\
\hline${ }^{64} \mathrm{Ni}_{g . s}\left(0^{+}\right)$ & $\left(2 p_{3 / 2}\right)$ & ${ }^{65} \mathrm{Ni}_{0.310}\left(3 / 2_{1}^{-}\right)$ & -0.147 \\
\hline${ }^{64} \mathrm{Ni}_{g . s}\left(0^{+}\right)$ & $\left(2 p_{3 / 2}\right)$ & ${ }^{65} \mathrm{Ni}_{0.693}\left(3 / 2_{2}^{-}\right)$ & 0.294 \\
\hline${ }^{64} \mathrm{Ni}_{g . s}\left(0^{+}\right)$ & $\left(1 g_{9 / 2}\right)$ & ${ }^{65} \mathrm{Ni}_{1.017}\left(9 / 2_{1}^{+}\right)$ & -0.670 \\
\hline${ }^{64} \mathrm{Ni}_{g . s}\left(0^{+}\right)$ & $\left(1 f_{5 / 2}\right)$ & ${ }^{65} \mathrm{Ni}_{1.27}(5 / 2-)$ & 0.130 \\
\hline${ }^{64} \mathrm{Ni}_{g . s}\left(0^{+}\right)$ & $\left(2 p_{1 / 2}\right)$ & ${ }^{65} \mathrm{Ni}_{1.41}\left(1 / 2_{2}^{-}\right)$ & 0.441 \\
\hline${ }^{64} \mathrm{Ni}_{1.345}\left(2^{+}\right)$ & $\begin{array}{l}\left(1 f_{5 / 2}\right) \\
\left(2 p_{3 / 2}\right) \\
\left(2 p_{1 / 2}\right)\end{array}$ & ${ }^{65} \mathrm{Ni}_{g . s}\left(5 / 2_{1}^{-}\right)$ & $\begin{array}{c}0.429 \\
0.266 \\
0.210\end{array}$ \\
\hline${ }^{64} \mathrm{Ni}_{1.345}\left(2^{+}\right)$ & $\begin{array}{l}\left(1 f_{5 / 2}\right) \\
\left(2 p_{3 / 2}\right)\end{array}$ & ${ }^{65} \mathrm{Ni}_{0.063}\left(1 / 2_{1}^{-}\right)$ & $\begin{array}{c}0.365 \\
0.503\end{array}$ \\
\hline${ }^{64} \mathrm{Ni}_{1.345}\left(2^{+}\right)$ & $\begin{array}{l}\left(1 f_{5 / 2}\right) \\
\left(2 p_{3 / 2}\right) \\
\left(2 p_{1 / 2}\right)\end{array}$ & ${ }^{65} \mathrm{Ni}_{0.310}\left(3 / 2_{1}^{-}\right)$ & $\begin{array}{c}0.958 \\
0.119 \\
0.022\end{array}$ \\
\hline${ }^{64} \mathrm{Ni}_{1.345}\left(2^{+}\right)$ & $\begin{array}{l}\left(1 f_{5 / 2}\right) \\
\left(2 p_{3 / 2}\right) \\
\left(2 p_{1 / 2}\right)\end{array}$ & ${ }^{65} \mathrm{Ni}_{0.693}\left(3 / 2_{2}^{-}\right)$ & $\begin{array}{r}-0.089 \\
0.296 \\
-0.170\end{array}$ \\
\hline${ }^{64} \mathrm{Ni}_{1.345}\left(2^{+}\right)$ & $\left(1 g_{9 / 2}\right)$ & ${ }^{65} \mathrm{Ni}_{1.017}\left(9 / 2_{1}^{+}\right)$ & -0.674 \\
\hline${ }^{64} \mathrm{Ni}_{1.345}\left(2^{+}\right)$ & $\begin{array}{l}\left(1 f_{5 / 2}\right) \\
\left(2 p_{3 / 2}\right) \\
\left(2 p_{1 / 2}\right)\end{array}$ & ${ }^{65} \mathrm{Ni}_{1.27}\left(5 / 2_{2}^{-}\right)$ & $\begin{array}{c}0.011 \\
0.181 \\
0.358\end{array}$ \\
\hline${ }^{64} \mathrm{Ni}_{1.345}\left(2^{+}\right)$ & $\begin{array}{l}\left(1 f_{5 / 2}\right) \\
\left(2 p_{3 / 2}\right)\end{array}$ & ${ }^{65} \mathrm{Ni}_{1.41}\left(1 / 2_{2}^{-}\right)$ & $\begin{array}{c}0.627 \\
0.117\end{array}$ \\
\hline${ }^{65} \mathrm{Ni}_{\text {g.s }}\left(5 / 2_{1}^{-}\right)$ & $\left(1 f_{5 / 2}\right)$ & ${ }^{66} \mathrm{Ni}_{g . s}\left(0_{1}^{+}\right)$ & 1.963 \\
\hline${ }^{65} \mathrm{Ni}_{g . s}\left(5 / 2_{1}^{-}\right)$ & $\begin{array}{l}\left(1 f_{5 / 2}\right) \\
\left(2 p_{3 / 2}\right) \\
\left(2 p_{1 / 2}\right)\end{array}$ & ${ }^{66} \mathrm{Ni}_{1.424}\left(2_{1}^{+}\right)$ & $\begin{array}{c}-0.025 \\
-0.156 \\
-0.534\end{array}$ \\
\hline${ }^{65} \mathrm{Ni}_{g . s}\left(5 / 2_{1}^{-}\right)$ & $\left(1 f_{5 / 2}\right)$ & ${ }^{66} \mathrm{Ni}_{2.445}\left(0_{2}^{+}\right)$ & -0.374 \\
\hline${ }^{65} \mathrm{Ni}_{g . s}\left(5 / 2_{1}^{-}\right)$ & $\begin{array}{l}\left(1 f_{5 / 2}\right) \\
\left(2 p_{3 / 2}\right) \\
\left(2 p_{1 / 2}\right)\end{array}$ & ${ }^{66} \mathrm{Ni}_{2.916}\left(2_{2}^{+}\right)$ & $\begin{array}{c}-0.176 \\
-0.039 \\
0.070\end{array}$ \\
\hline${ }^{65} \mathrm{Ni}_{0.063}\left(1 / 2_{1}^{-}\right)$ & $\left(2 p_{1 / 2}\right)$ & ${ }^{66} \mathrm{Ni}_{g . s}\left(0_{1}^{+}\right)$ & 0.617 \\
\hline${ }^{65} \mathrm{Ni}_{0.063}\left(1 / 2_{1}^{-}\right)$ & $\begin{array}{l}\left(1 f_{5 / 2}\right) \\
\left(2 p_{3 / 2}\right)\end{array}$ & ${ }^{66} \mathrm{Ni}_{1.424}\left(2_{1}^{+}\right)$ & $\begin{array}{l}-0.461 \\
-0.328\end{array}$ \\
\hline${ }^{65} \mathrm{Ni}_{0.063}\left(1 / 2_{1}^{-}\right)$ & $\left(2 p_{1 / 2}\right)$ & ${ }^{66} \mathrm{Ni}_{2.445}\left(0_{2}^{+}\right)$ & 0.685 \\
\hline${ }^{65} \mathrm{Ni}_{0.063}\left(1 / 2_{1}^{-}\right)$ & $\begin{array}{l}\left(1 f_{5 / 2}\right) \\
\left(2 p_{3 / 2}\right)\end{array}$ & ${ }^{66} \mathrm{Ni}_{2.916}\left(2_{2}^{+}\right)$ & $\begin{array}{r}-0.029 \\
0.055\end{array}$ \\
\hline${ }^{65} \mathrm{Ni}_{0.310}\left(3 / 2_{1}^{-}\right)$ & $\left(2 p_{3 / 2}\right)$ & ${ }^{66} \mathrm{Ni}_{g . s}\left(0_{1}^{+}\right)$ & -0.354 \\
\hline${ }^{65} \mathrm{Ni}_{0.310}\left(3 / 2_{1}^{-}\right)$ & $\begin{array}{l}\left(1 f_{5 / 2}\right) \\
\left(2 p_{3 / 2}\right) \\
\left(2 p_{1 / 2}\right)\end{array}$ & ${ }^{66} \mathrm{Ni}_{1.424}\left(2_{1}^{+}\right)$ & $\begin{array}{c}-0.914 \\
0.306 \\
0.212\end{array}$ \\
\hline${ }^{65} \mathrm{Ni}_{0.310}\left(3 / 2_{1}^{-}\right)$ & $\left(2 p_{3 / 2}\right)$ & ${ }^{66} \mathrm{Ni}_{2.445}\left(0_{2}^{+}\right)$ & 0.113 \\
\hline${ }^{65} \mathrm{Ni}_{0.310}\left(3 / 2_{1}^{-}\right)$ & $\begin{array}{l}\left(1 f_{5 / 2}\right) \\
\left(2 p_{3 / 2}\right) \\
\left(2 p_{1 / 2}\right)\end{array}$ & ${ }^{66} \mathrm{Ni}_{2.916}\left(2_{2}^{+}\right)$ & $\begin{array}{r}-0.155 \\
-0.044 \\
0.193\end{array}$ \\
\hline
\end{tabular}

subspace are identical to the matrix elements in the bosonic space. As a first approach one can consider the simple
TABLE III. Table II continued.

\begin{tabular}{|c|c|c|c|}
\hline Initial state & $j$ & Final state & Spect. ampl. \\
\hline${ }^{65} \mathrm{Ni}_{0.693}\left(3 / 2_{2}^{-}\right)$ & $\begin{array}{l}\left(2 p_{3 / 2}\right) \\
\left(1 f_{5 / 2}\right)\end{array}$ & ${ }^{66} \mathrm{Ni}_{g . s}\left(0_{1}^{+}\right)$ & $\begin{array}{r}1.575 \\
-0.164\end{array}$ \\
\hline${ }^{65} \mathrm{Ni}_{0.693}\left(3 / 2_{2}^{-}\right)$ & $\begin{array}{l}\left(2 p_{3 / 2}\right) \\
\left(2 p_{1 / 2}\right)\end{array}$ & ${ }^{66} \mathrm{Ni}_{1.424}\left(2_{1}^{+}\right)$ & $\begin{array}{r}0.334 \\
-0.169\end{array}$ \\
\hline${ }^{65} \mathrm{Ni}_{0.693}\left(3 / 2_{2}^{-}\right)$ & $\begin{array}{l}\left(2 p_{3 / 2}\right) \\
\left(1 f_{5 / 2}\right)\end{array}$ & ${ }^{66} \mathrm{Ni}_{2.445}\left(0_{2}^{+}\right)$ & $\begin{array}{r}0.038 \\
-0.015\end{array}$ \\
\hline${ }^{65} \mathrm{Ni}_{0.693}\left(3 / 2_{2}^{-}\right)$ & $\begin{array}{l}\left(2 p_{3 / 2}\right) \\
\left(2 p_{1 / 2}\right)\end{array}$ & ${ }^{66} \mathrm{Ni}_{2.916}\left(2_{2}^{+}\right)$ & $\begin{array}{l}-0.026 \\
-0.123\end{array}$ \\
\hline${ }^{65} \mathrm{Ni}_{1.017}\left(9 / 2_{1}^{+}\right)$ & $\left(1 g_{9 / 2}\right)$ & ${ }^{66} \mathrm{Ni}_{g . s}\left(0_{1}^{+}\right)$ & -0.258 \\
\hline${ }^{65} \mathrm{Ni}_{1.017}\left(9 / 2_{1}^{+}\right)$ & $\left(1 g_{9 / 2}\right)$ & ${ }^{66} \mathrm{Ni}_{1.424}\left(2_{1}^{+}\right)$ & 0.171 \\
\hline${ }^{65} \mathrm{Ni}_{1.017}\left(9 / 2_{1}^{+}\right)$ & $\left(1 g_{9 / 2}\right)$ & ${ }^{66} \mathrm{Ni}_{2.445}\left(0_{2}^{+}\right)$ & 0.680 \\
\hline${ }^{65} \mathrm{Ni}_{1.017}\left(9 / 2_{1}^{+}\right)$ & $\left(1 g_{9 / 2}\right)$ & ${ }^{66} \mathrm{Ni}_{2.916}\left(2_{2}^{+}\right)$ & -1.063 \\
\hline${ }^{65} \mathrm{Ni}_{1.27}\left(5 / 22_{2}^{-}\right)$ & $\begin{array}{l}\left(1 f_{5 / 2}\right) \\
\left(1 f_{5 / 2}\right)\end{array}$ & ${ }^{66} \mathrm{Ni}_{g . s}\left(0_{1}^{+}\right)$ & $\begin{array}{l}-0.070 \\
-0.604\end{array}$ \\
\hline${ }^{65} \mathrm{Ni}_{1.27}\left(5 / 2_{2}^{-}\right)$ & $\begin{array}{l}\left(2 p_{3 / 2}\right) \\
\left(2 p_{1 / 2}\right)\end{array}$ & ${ }^{66} \mathrm{Ni}_{1.424}\left(2_{1}^{+}\right)$ & $\begin{array}{r}-0.517 \\
0.457\end{array}$ \\
\hline${ }^{65} \mathrm{Ni}_{1.27}\left(5 / 2_{2}^{-}\right)$ & $\begin{array}{l}\left(1 f_{5 / 2}\right) \\
\left(1 f_{5 / 2}\right)\end{array}$ & ${ }^{66} \mathrm{Ni}_{2.445}\left(0_{2}^{+}\right)$ & $\begin{array}{r}0.934 \\
-0.195\end{array}$ \\
\hline${ }^{65} \mathrm{Ni}_{1.27}\left(5 / 2_{2}^{-}\right)$ & $\begin{array}{l}\left(2 p_{3 / 2}\right) \\
\left(2 p_{1 / 2}\right)\end{array}$ & ${ }^{66} \mathrm{Ni}_{2.916}\left(2_{2}^{+}\right)$ & $\begin{array}{r}-0.025 \\
0.068\end{array}$ \\
\hline${ }^{65} \mathrm{Ni}_{1.41}\left(1 / 2_{2}^{-}\right)$ & $\left(2 p_{1 / 2}\right)$ & ${ }^{66} \mathrm{Ni}_{g . s}\left(0_{1}^{+}\right)$ & 0.482 \\
\hline${ }^{65} \mathrm{Ni}_{1.41}\left(1 / 2_{2}^{-}\right)$ & $\begin{array}{l}\left(1 f_{5 / 2}\right) \\
\left(2 p_{3 / 2}\right)\end{array}$ & ${ }^{66} \mathrm{Ni}_{1.424}\left(2_{1}^{+}\right)$ & $\begin{array}{l}0.010 \\
0.474\end{array}$ \\
\hline${ }^{65} \mathrm{Ni}_{1.41}\left(1 / 2_{2}^{-}\right)$ & $\left(2 p_{1 / 2}\right)$ & ${ }^{66} \mathrm{Ni}_{2.445}\left(0_{2}^{+}\right)$ & 0.421 \\
\hline${ }^{65} \mathrm{Ni}_{1.41}\left(1 / 2_{2}^{-}\right)$ & $\begin{array}{l}\left(1 f_{5 / 2}\right) \\
\left(2 p_{3 / 2}\right)\end{array}$ & ${ }^{66} \mathrm{Ni}_{2.916}\left(2_{2}^{+}\right)$ & $\begin{array}{r}-0.017 \\
0.073\end{array}$ \\
\hline
\end{tabular}

mapping,

$$
\begin{gathered}
\left(\rho_{j}^{\dagger} \rho_{j^{\prime}}^{\dagger}\right)_{M}^{(0)} \rightarrow A_{\rho}\left(j, j^{\prime}\right)\left(s_{\rho}^{\dagger}\right), \\
\left(\rho_{j}^{\dagger} \rho_{j^{\prime}}^{\dagger}\right)_{M}^{(2)} \rightarrow B_{\rho}\left(j, j^{\prime}\right)\left(d_{\rho}^{\dagger}\right)_{M},
\end{gathered}
$$

where the label $\rho$ refers to neutron $(n)$ or proton $(p)$. The coefficients $A_{\rho}\left(j, j^{\prime}\right)$ and $B_{\rho}\left(j, j^{\prime}\right)$ are taken from Ref. [48].

The bosonization method, when carried to all orders, produces results that are identical to the fermionic results. To investigate the extent to which our calculations are reliable, we have carried out the OAI expansion to next to leading order (NLO),

$$
\begin{aligned}
\left(\rho_{j}^{\dagger} \rho_{j^{\prime}}^{\dagger}\right)_{M}^{(2)} & \rightarrow B_{\rho}\left(j, j^{\prime}\right)\left(d_{\rho}^{\dagger}\right)_{M}+C_{\rho}\left(j, j^{\prime}\right) s_{\rho}^{\dagger}\left(s_{\rho}^{\dagger} \tilde{d}_{\rho}\right)_{M}^{(2)} \\
& +D_{\rho}\left(j, j^{\prime}\right) s_{\rho}^{\dagger}\left(d_{\rho}^{\dagger} \tilde{d}_{\rho}\right)_{M}^{(2)} .
\end{aligned}
$$

The coefficients $C_{\rho}\left(j, j^{\prime}\right)$ and $D_{\rho}\left(j, j^{\prime}\right)$ are taken from Ref. [48]. A boson expansion of the pair operators up to the second term in Eq. (5) was carried out many years ago [34], with the purpose of calculating $2 v$ double- $\beta$ decay matrix elements in the closure approximation.

The microscopic IBM-2 spectroscopic factors contain the effective microscopic interaction about the valence shells, providing a more realistic result for the nucleon transfer reaction calculations. We consider a generalization of the exact theory for nondegenerate orbits [52]. The structure constants [53], $\alpha_{j}$ and $\beta_{j, j^{\prime}}$, that we considered depend on 


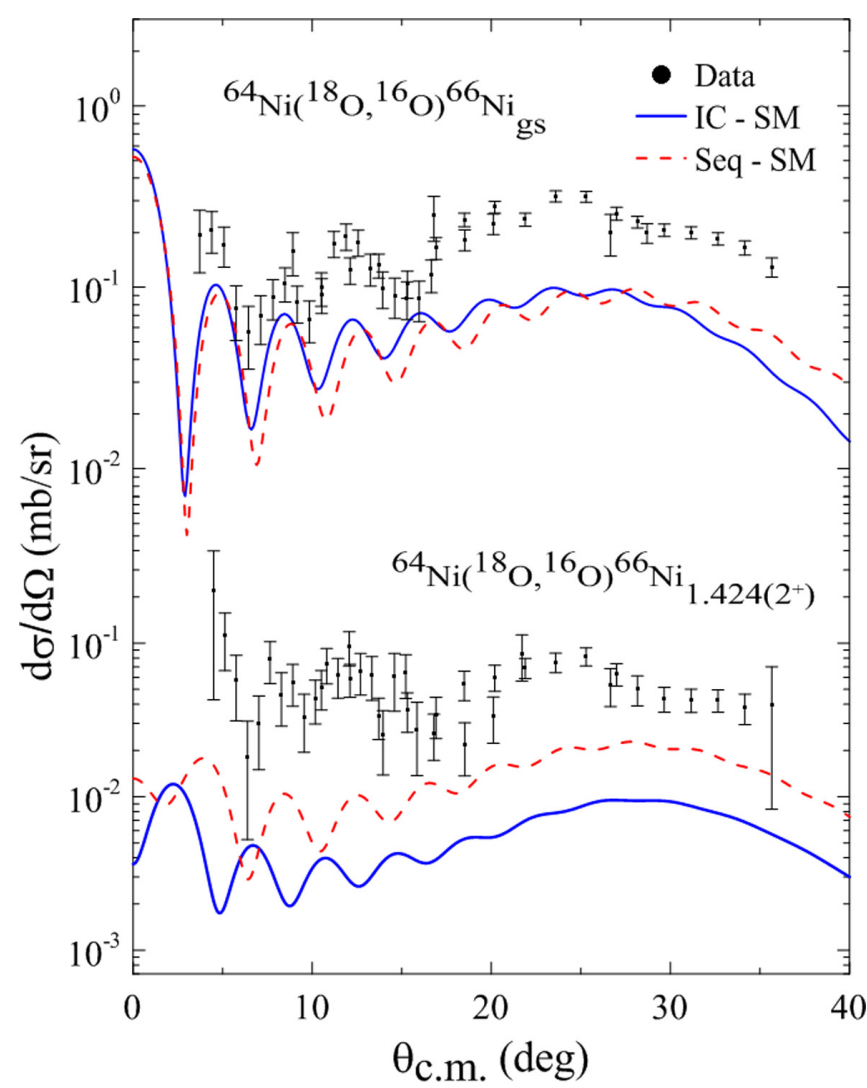

FIG. 4. Comparison of the experiment angular distribution with the independent coordinate and two-step DWBA calculations for the reaction ${ }^{64} \mathrm{Ni}\left({ }^{18} \mathrm{O},{ }^{16} \mathrm{O}\right){ }^{66} \mathrm{Ni}$. The spectroscopic amplitudes from Tables I, II, and III are used.

the single-particle energies for the neutrons as particles in the 28-50 neutron shell with four valence orbits: $1 f_{5 / 2}, 2 p_{3 / 2}$, $2 p_{1 / 2}$, and $1 g_{9 / 2}$.

The pair structure coefficients [53], $\alpha_{j}$ and $\beta_{j, j^{\prime}}$, are reported in Table $\mathrm{V}$ and they were considered to depend on the single-particle energies.

The single-particle energies were obtained by solving a Schrodinger equation that includes a Woods-Saxon potential (more details will be presented elsewhere [54]) with a typical set of parameters $V_{0}=53-\mathrm{MeV}, V_{1}=-30 \mathrm{MeV}$, and $V_{s o}=$ $22 \mathrm{MeV}$ for the strengths, and $r_{0}=r_{s o}=1.25 \mathrm{fm}$ and $a_{0}=$ $a_{s 0}=0.65 \mathrm{fm}$ for the geometry. For the Coulomb term the radius is a little smaller with $r_{c}=1.20 \mathrm{fm}$.

In our calculations we modify the central potential strength $V_{o}^{\prime}=V_{N} V_{o}$ and $a_{o}$ and $r_{o}$ are changed to $a_{o}^{\prime}=0.7 \mathrm{fm}$ and $r_{o}^{\prime}=1.3 \mathrm{fm}$.

TABLE IV. Coefficients used in the microscopic IBM-2 calculations.

\begin{tabular}{lcccccrc}
\hline \hline Nucleus & $N_{v}$ & $N_{\pi}$ & $\begin{array}{c}\epsilon_{d} \\
(\mathrm{MeV})\end{array}$ & $\begin{array}{c}c_{v}^{(0)} \\
(\mathrm{MeV})\end{array}$ & $\begin{array}{c}c_{v}^{(2)} \\
(\mathrm{MeV})\end{array}$ & $\begin{array}{c}c_{v}^{(4)} \\
(\mathrm{MeV})\end{array}$ & $\begin{array}{c}\mathrm{v}_{l n} \\
(\mathrm{MeV})\end{array}$ \\
\hline${ }^{64} \mathrm{Ni}$ & 4 & 0 & 1.2 & 0.62 & -0.27 & -0.274 & 0.0242 \\
${ }^{66} \mathrm{Ni}$ & 5 & 0 & 1.2 & 0.45 & 0.29 & 0.036 & 0.0374 \\
\hline \hline
\end{tabular}
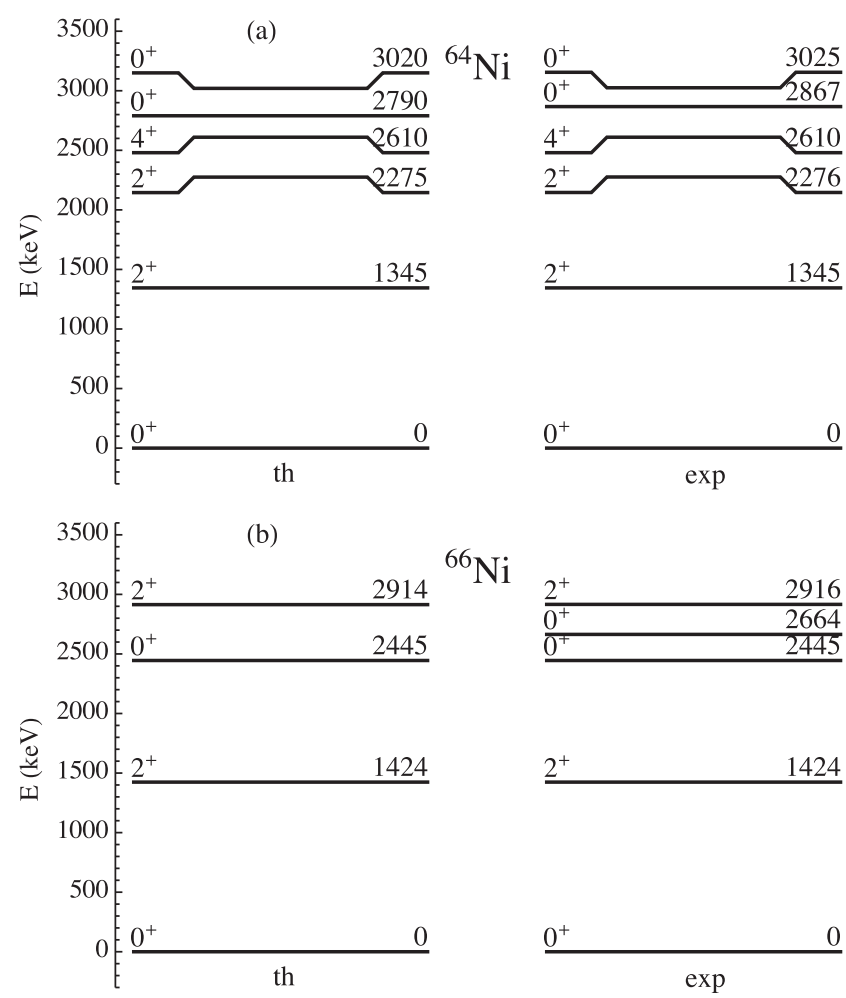

FIG. 5. Comparison between calculated and experimental lowlying spectra for the ${ }^{64} \mathrm{Ni}$ and ${ }^{66} \mathrm{Ni}$ isotopes.

Following the previous considerations the neutrons in the 28-50 neutron shell have the single-particle energies: $e_{5 / 2}=2.788 \mathrm{MeV}, e_{3 / 2}=0.0 \mathrm{MeV}, e_{1 / 2}=2.391 \mathrm{MeV}$, and $e_{9 / 2}=4.027 \mathrm{MeV}$ and for the microscopic mapping we consider the surface delta interaction with strength $A=0.366$.

\section{IBFM-2, sequential calculation}

In the previous section we have presented the description of one-step calculation of the two-neutron transfer reaction within the microscopic IBM-2, where a neutron boson is transferred. Here, we present the formalism to describe the two-step transfer reaction (sequentially). In this case the intermediate states of the odd-even ${ }^{65} \mathrm{Ni}$ nucleus should be considered as shown in Fig. 2. To describe the odd-even nucleus we have to use the formalism of the interacting boson fermion model 2 (IBFM-2) [23], which takes into account the degrees of freedom of one single fermion.

To construct the odd-even ${ }^{65} \mathrm{Ni}$ nucleus, i.e., the core nucleus plus an extra neutron, the odd-even nuclei are described in the context of the IBFM-2 by the Hamiltonian,

$$
H=H^{B}+H_{\rho}^{F}+V_{\rho}^{\mathrm{BF}},
$$

where $H^{B}$ is the boson Hamiltonian that describes the core nucleus and the label $\rho$ refers to the $p$ (extra proton) or $n$ (extra neutron) added to the even-even core to form the odd-even nucleus. $H_{\rho}^{F}$ is the single-particle Hamiltonian that describes the extra nucleon. It is written as

$$
H_{\rho}^{F}=\sum_{j_{\rho}} \epsilon_{j_{\rho}} \hat{n}_{j_{\rho}},
$$


TABLE V. Pair structure coefficients $\alpha_{j}$ and $\beta_{j, j^{\prime}}$ in the $N, Z=28-50$ shell.

\begin{tabular}{lc}
\hline \hline & Neutrons (particles) \\
\hline$\alpha_{1 / 2}$ & 0.461 \\
$\alpha_{3 / 2}$ & 2.218 \\
$\alpha_{5 / 2}$ & 0.408 \\
$\alpha_{9 / 2}$ & -0.299 \\
$\beta_{1 / 2,3 / 2}$ & -0.117 \\
$\beta_{3 / 2,3 / 2}$ & 0.986 \\
$\beta_{1 / 2,5 / 2}$ & 0.069 \\
$\beta_{5 / 2,5 / 2}$ & -0.049 \\
$\beta_{9 / 2,9 / 2}$ & 0.045 \\
\hline \hline
\end{tabular}

where $\epsilon_{j_{\rho}}$ is the quasi-single-particle energy of the extra nucleon, and $\hat{n}$ is the number operator. The quasiparticle energies $\epsilon_{j_{\rho}}$ are obtained within the BCS approximation with a gap $\Delta=12 / \sqrt{A}$, where $A$ is the mass number of the even-even core nucleus. In the BCS approximation, the quasiparticle energy is related to the single-particle level $\epsilon^{s p}$, the occupation probabilities $v_{j}$, and the Fermi level $\lambda$. The single-particle energies were obtained solving the Woods-Saxon potential. For the ${ }^{64} \mathrm{Ni}$ nucleus it is taken a diffuseness $a_{o}^{\prime}=0.7 \mathrm{fm}$ and reduced radius $r_{o}^{\prime}=1.3 \mathrm{fm}$ and for the ${ }^{66} \mathrm{Ni}$ isotope $a_{o}^{\prime}=0.65 \mathrm{fm}$ and reduced radius $r_{o}^{\prime}=1.25 \mathrm{fm}$ [54]. The last term in Eq. (6) is the boson-fermion interaction $V_{\mathrm{BF}}$. It arises from a quadrupole force between protons and neutrons, and it depends on the three parameters reported in Table VI.

We computed the spectroscopic amplitudes of one-neutron transfer reactions between ${ }^{64} \mathrm{Ni} \rightarrow{ }^{65} \mathrm{Ni}$, and ${ }^{65} \mathrm{Ni} \rightarrow{ }^{66} \mathrm{Ni}$. The even-even nuclei ${ }^{64} \mathrm{Ni}$ and ${ }^{66} \mathrm{Ni}$ are described within the microscopic IBM-2, as in the previous section, and the odd-even ${ }^{65} \mathrm{Ni}$ nucleus is described by the IBFM-2. The ${ }^{65} \mathrm{Ni}$ nucleus may be constructed in two different ways, the first as ${ }^{64} \mathrm{Ni}$ plus a neutron particle or a second option is ${ }^{66} \mathrm{Ni}$ plus a neutron hole. The parameters used to reproduce the experimental energies are reported in Table VI. Our theoretical spectrum is presented in Fig. 6 in comparison with the experimental data. The spectroscopic amplitudes calculated within the IBFM-2 are presented in Tables VIII and IX.

In Fig. 7 the comparison of the same experimental twoneutron transfer reaction cross section shown in Fig. 4 with the theoretical results for the direct and sequential mechanisms, but using the spectroscopic amplitudes derived using the microscopic IBM-2 and the IBFM-2 are shown.

TABLE VI. Coefficients used in the IBFM-2 calculations. Set I corresponds to the neutron particle coupled to the core ${ }^{64} \mathrm{Ni}$ and set II corresponds to the coupling of the neutron hole to the core ${ }^{66} \mathrm{Ni}$.

\begin{tabular}{lccc}
\hline \hline Core & $\begin{array}{c}\Gamma_{\nu v} \\
(\mathrm{MeV})\end{array}$ & $\begin{array}{c}\chi_{v} \\
(\mathrm{MeV})\end{array}$ & $\begin{array}{c}A_{\nu v} \\
(\mathrm{MeV})\end{array}$ \\
\hline${ }^{64} \mathrm{Ni}$ & -1.15 & 5.982 & 0.69 \\
${ }^{66} \mathrm{Ni}$ & -1.15 & 5.982 & 0.74 \\
\hline \hline
\end{tabular}

TABLE VII. Spectroscopic amplitudes used in the CRC calculations for two-neutron transfer using the microscopic IBM-2 method. $j_{1} j_{2}$ are the spins of the neutron orbitals for two-neutron transfer, and $J_{12}$ is the angular momentum of the two-neutron system.

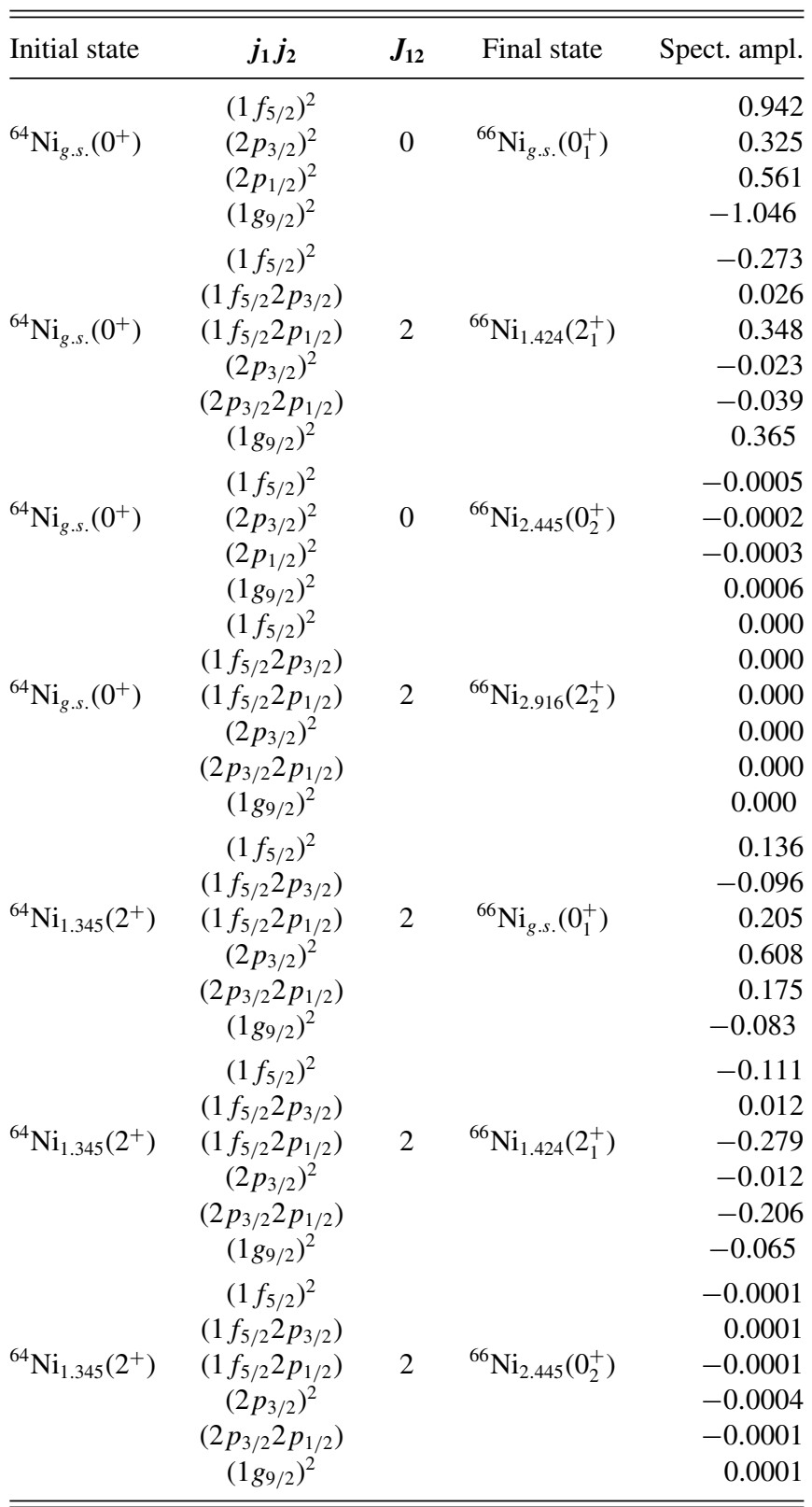

\section{E. Discussion}

The extreme cluster model calculations described in Sec. III A overestimates the experimental data as shown in Fig. 3. This could indicate that this approximation is not suitable for the calculations of target overlaps, because it oversimplifies its nuclear structure. This is especially relevant for the two-neutron transfer to the first $2^{+}$excited state of ${ }^{66} \mathrm{Ni}$ [Fig. 3(b)], where the experimental value is overestimated of about one order of magnitude. However, this model can be used to extract spectroscopic information in an approximate way. This was done in Ref. [3], where the spectroscopic amplitudes derived by scaling the calculations on the data were 


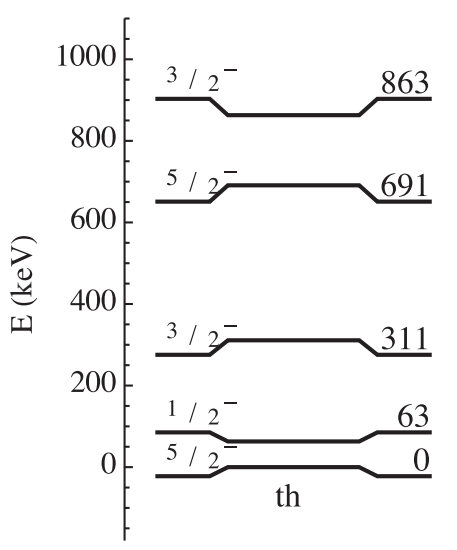

${ }^{65} \mathrm{Ni}$

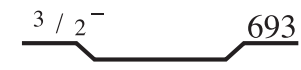

FIG. 6. Comparison between calculated and experimental lowlying spectra for nucleus ${ }^{65} \mathrm{Ni}$ using the set I of parameters.

in reasonable agreement with the corresponding shell-model component for that overlap. This procedure was applied also here. The resulting spectroscopic amplitude for the overlap of the ground states of the ${ }^{64} \mathrm{Ni}$ and ${ }^{66} \mathrm{Ni}$ nuclei was found 0.71. For the $<{ }^{64} \mathrm{NiO}^{+} \mid{ }^{66} \mathrm{Ni} 2^{+}>$overlap, it was found 0.37 . The shell-model values from Table II for the corresponding overlaps are 0.90 and 0.22 . This shows that the extreme cluster

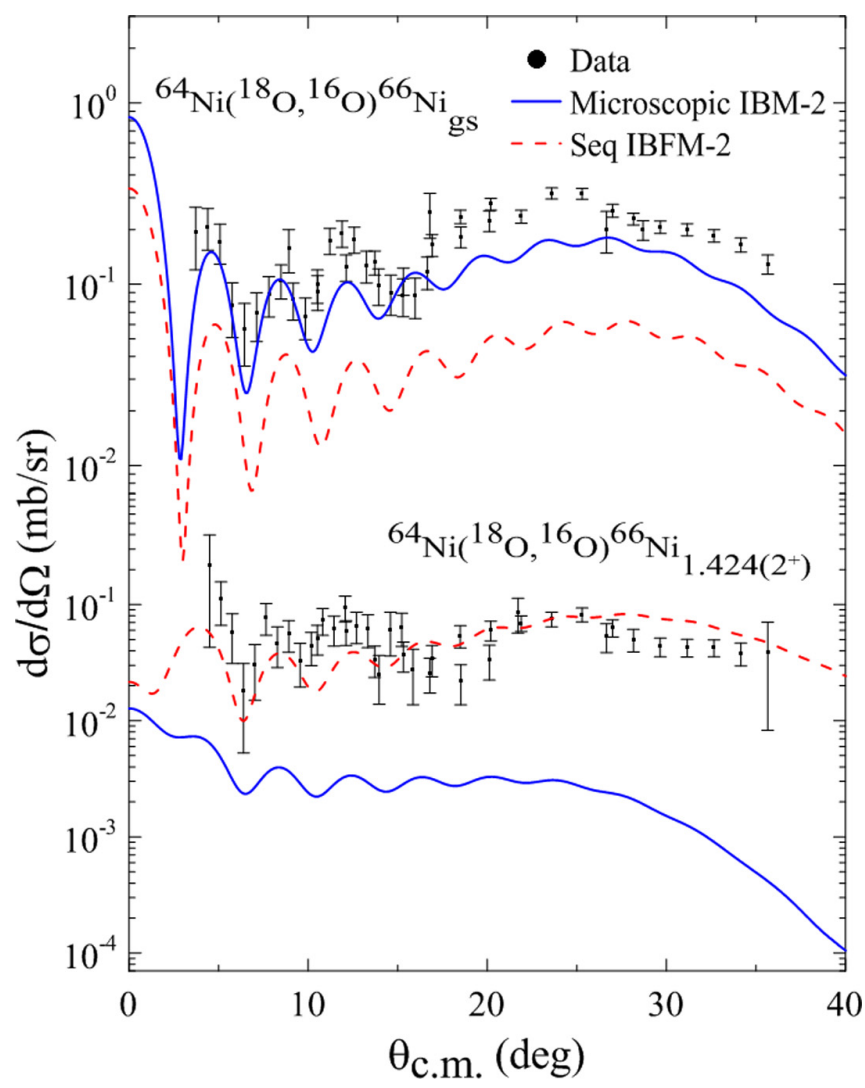

FIG. 7. Comparison of the experiment angular distribution with the independent coordinate and two-step DWBA calculations for the reaction ${ }^{64} \mathrm{Ni}\left({ }^{18} \mathrm{O},{ }^{16} \mathrm{O}\right){ }^{66} \mathrm{Ni}$. The spectroscopic amplitudes from Tables VII, VIII, and IX were used.
TABLE VIII. Spectroscopic amplitudes used in the DWBA calculations for one-neutron transfer using using the IBFM-2 method, where $j$ is the spin of the neutron orbitals.

\begin{tabular}{|c|c|c|c|}
\hline Initial state & $j$ & Final state & Spect. ampl. \\
\hline${ }^{64} \mathrm{Ni}_{g . s}\left(0^{+}\right)$ & $\left(1 f_{5 / 2}\right)$ & ${ }^{65} \mathrm{Ni}_{g . s}\left(5 / 2_{1}^{-}\right)$ & -1.446 \\
\hline${ }^{64} \mathrm{Ni}_{g . s}\left(0^{+}\right)$ & $\left(2 p_{1 / 2}\right)$ & ${ }^{65} \mathrm{Ni}_{0.063}\left(1 / 2_{1}^{-}\right)$ & 0.981 \\
\hline${ }^{64} \mathrm{Ni}_{g . s}\left(0^{+}\right)$ & $\left(2 p_{3 / 2}\right)$ & ${ }^{65} \mathrm{Ni}_{0.310}\left(3 / 2_{1}^{-}\right)$ & 0.181 \\
\hline${ }^{64} \mathrm{Ni}_{g . s}\left(0^{+}\right)$ & $\left(2 p_{3 / 2}\right)$ & ${ }^{65} \mathrm{Ni}_{0.693}\left(3 / 2_{2}^{-}\right)$ & -0.258 \\
\hline${ }^{64} \mathrm{Ni}_{g . s}\left(0^{+}\right)$ & $\left(1 f_{5 / 2}\right)$ & ${ }^{65} \mathrm{Ni}_{1.27}\left(5 / 22_{2}^{-}\right)$ & 1.016 \\
\hline \multirow[t]{2}{*}{${ }^{64} \mathrm{Ni}_{g . s}\left(0^{+}\right)$} & $\left(2 p_{1 / 2}\right)$ & ${ }^{65} \mathrm{Ni}_{1.41}\left(1 / 2_{2}^{-}\right)$ & -0.059 \\
\hline & $\left(1 f_{5 / 2}\right)$ & & -0.629 \\
\hline \multirow[t]{2}{*}{${ }^{64} \mathrm{Ni}_{1.345}\left(2^{+}\right)$} & $\left(2 p_{3 / 2}\right)$ & ${ }^{65} \mathrm{Ni}_{g . s}\left(5 / 2_{1}^{-}\right)$ & 0.082 \\
\hline & $\left(2 p_{1 / 2}\right)$ & & 0.447 \\
\hline \multirow[t]{3}{*}{${ }^{64} \mathrm{Ni}_{1.345}\left(2^{+}\right)$} & $\left(1 f_{5 / 2}\right)$ & ${ }^{65} \mathrm{Ni}_{0.063}\left(1 / 2_{1}^{-}\right)$ & -0.946 \\
\hline & $\left(2 p_{3 / 2}\right)$ & & 0.446 \\
\hline & $\left(1 f_{5 / 2}\right)$ & & -0.355 \\
\hline \multirow{3}{*}{${ }^{64} \mathrm{Ni}_{1.345}\left(2^{+}\right)$} & $\left(2 p_{3 / 2}\right)$ & ${ }^{65} \mathrm{Ni}_{0.310}\left(3 / 2_{1}^{-}\right)$ & -0.031 \\
\hline & $\left(2 p_{1 / 2}\right)$ & & -0.416 \\
\hline & $\left(1 f_{5 / 2}\right)$ & & 0.069 \\
\hline \multirow{3}{*}{${ }^{64} \mathrm{Ni}_{1.345}\left(2^{+}\right)$} & $\left(2 p_{3 / 2}\right)$ & ${ }^{65} \mathrm{Ni}_{0.693}\left(3 / 2_{2}^{-}\right)$ & -0.035 \\
\hline & $\left(2 p_{1 / 2}\right)$ & & 0.280 \\
\hline & $\left(1 f_{5 / 2}\right)$ & & 0.199 \\
\hline \multirow[t]{2}{*}{${ }^{64} \mathrm{Ni}_{1.345}\left(2^{+}\right)$} & $\left(2 p_{3 / 2}\right)$ & ${ }^{65} \mathrm{Ni}_{1.27}\left(5 / 2_{2}^{-}\right)$ & -0.341 \\
\hline & $\left(2 p_{1 / 2}\right)$ & & 0.057 \\
\hline \multirow[t]{2}{*}{${ }^{65} \mathrm{Ni}_{g . s}\left(5 / 2_{1}^{-}\right)$} & $\left(1 f_{5 / 2}\right)$ & ${ }^{66} \mathrm{Ni}_{g . s}\left(0_{1}^{+}\right)$ & -0.949 \\
\hline & $\left(1 f_{5 / 2}\right)$ & & 0.398 \\
\hline \multirow{2}{*}{${ }^{65} \mathrm{Ni}_{g . s}\left(5 / 2_{1}^{-}\right)$} & $\left(2 p_{3 / 2}\right)$ & ${ }^{66} \mathrm{Ni}_{1.424}\left(2_{1}^{+}\right)$ & -0.991 \\
\hline & $\left(2 p_{1 / 2}\right)$ & & -0.149 \\
\hline \multirow{2}{*}{${ }^{65} \mathrm{Ni}_{g . s}\left(5 / 2_{1}^{-}\right)$} & $\left(1 f_{5 / 2}\right)$ & ${ }^{66} \mathrm{Ni}_{2.445}\left(0_{2}^{+}\right)$ & -0.106 \\
\hline & $\left(1 f_{5 / 2}\right)$ & & 0.042 \\
\hline \multirow{2}{*}{${ }^{65} \mathrm{Ni}_{g . s}\left(5 / 21_{1}^{-}\right)$} & $\left(2 p_{3 / 2}\right)$ & ${ }^{66} \mathrm{Ni}_{2.916}\left(2_{2}^{+}\right)$ & -0.290 \\
\hline & $\left(2 p_{1 / 2}\right)$ & & 0.055 \\
\hline${ }^{65} \mathrm{Ni}_{0.063}\left(1 / 2_{1}^{-}\right)$ & $\left(2 p_{1 / 2}\right)$ & ${ }^{66} \mathrm{Ni}_{g . s}\left(0_{1}^{+}\right)$ & 0.665 \\
\hline \multirow[t]{2}{*}{${ }^{65} \mathrm{Ni}_{0.063}\left(1 / 2_{1}^{-}\right)$} & $\left(1 f_{5 / 2}\right)$ & ${ }^{66} \mathrm{Ni}_{1.424}\left(2_{1}^{+}\right)$ & 0.816 \\
\hline & $\left(2 p_{3 / 2}\right)$ & & -0.511 \\
\hline${ }^{65} \mathrm{Ni}_{0.063}\left(1 / 2_{1}^{-}\right)$ & $\left(2 p_{1 / 2}\right)$ & ${ }^{66} \mathrm{Ni}_{2.445}\left(\mathrm{O}_{2}^{+}\right)$ & -0.029 \\
\hline \multirow[t]{2}{*}{${ }^{65} \mathrm{Ni}_{0.063}\left(1 / 2_{1}^{-}\right)$} & $\left(1 f_{5 / 2}\right)$ & ${ }^{66} \mathrm{Ni}_{2.916}\left(2_{2}^{+}\right)$ & 0.004 \\
\hline & $\left(2 p_{3 / 2}\right)$ & & 0.103 \\
\hline \multirow[t]{2}{*}{${ }^{65} \mathrm{Ni}_{0.310}\left(3 / 2_{1}^{-}\right)$} & $\left(2 p_{3 / 2}\right)$ & ${ }^{66} \mathrm{Ni}_{g . s}\left(0_{1}^{+}\right)$ & 0.730 \\
\hline & $\left(1 f_{5 / 2}\right)$ & & -0.628 \\
\hline \multirow[t]{2}{*}{${ }^{65} \mathrm{Ni}_{0.310}\left(3 / 2_{1}^{-}\right)$} & $\left(2 p_{3 / 2}\right)$ & ${ }^{66} \mathrm{Ni}_{1.424}\left(2_{1}^{+}\right)$ & -1.017 \\
\hline & $\left(2 p_{1 / 2}\right)$ & & -0.058 \\
\hline \multirow[t]{2}{*}{${ }^{65} \mathrm{Ni}_{0.310}\left(3 / 2_{1}^{-}\right)$} & $\left(2 p_{3 / 2}\right)$ & ${ }^{66} \mathrm{Ni}_{2.445}\left(0_{2}^{+}\right)$ & 0.583 \\
\hline & $\left(1 f_{5 / 2}\right)$ & & -0.298 \\
\hline \multirow{2}{*}{${ }^{65} \mathrm{Ni}_{0.310}\left(3 / 2_{1}^{-}\right)$} & $\left(2 p_{3 / 2}\right)$ & ${ }^{66} \mathrm{Ni}_{2.916}\left(2_{2}^{+}\right)$ & -0.748 \\
\hline & $\left(2 p_{1 / 2}\right)$ & & -0.172 \\
\hline \multirow[t]{2}{*}{${ }^{65} \mathrm{Ni}_{0.693}\left(3 / 22_{2}^{-}\right)$} & $\left(2 p_{3 / 2}\right)$ & ${ }^{66} \mathrm{Ni}_{g . s}\left(0_{1}^{+}\right)$ & -1.062 \\
\hline & $\left(1 f_{5 / 2}\right)$ & & 0.408 \\
\hline \multirow[t]{2}{*}{${ }^{65} \mathrm{Ni}_{0.693}\left(3 / 2_{2}^{-}\right)$} & $\left(2 p_{3 / 2}\right)$ & ${ }^{66} \mathrm{Ni}_{1.424}\left(2_{1}^{+}\right)$ & 0.416 \\
\hline & $\left(2 p_{1 / 2}\right)$ & & -0.363 \\
\hline
\end{tabular}

model can be used to derive the approximate values of the spectroscopic amplitudes. 
TABLE IX. Table VIII continued.

\begin{tabular}{lccr}
\hline \hline Initial state & $j$ & Final state & Spect. ampl. \\
\hline${ }^{65} \mathrm{Ni}_{0.693}(3 / 2-)$ & $\left(2 p_{3 / 2}\right)$ & ${ }^{66} \mathrm{Ni}_{2.445}\left(0_{2}^{+}\right)$ & 0.716 \\
& $\left(1 f_{5 / 2}\right)$ & & -0.212 \\
${ }^{65} \mathrm{Ni}_{0.693}(3 / 2-)$ & $\left(2 p_{3 / 2}\right)$ & ${ }^{66} \mathrm{Ni}_{2.916}\left(2_{2}^{+}\right)$ & -0.766 \\
& $\left(2 p_{1 / 2}\right)$ & & 0.313 \\
${ }^{65} \mathrm{Ni}_{1.27}(5 / 2-)$ & $\left(1 f_{5 / 2}\right)$ & ${ }^{66} \mathrm{Ni}_{g . s}\left(0_{1}^{+}\right)$ & -0.639 \\
& $\left(1 f_{5 / 2}\right)$ & & 0.481 \\
${ }^{65} \mathrm{Ni}_{1.27}(5 / 2-)$ & $\left(2 p_{3 / 2}\right)$ & ${ }^{66} \mathrm{Ni}_{1.424}\left(2_{1}^{+}\right)$ & 1.129 \\
& $\left(2 p_{1 / 2}\right)$ & & -0.448 \\
${ }^{65} \mathrm{Ni}_{1.27}(5 / 2-)$ & $\left(1 g_{9 / 2}\right)$ & ${ }^{66} \mathrm{Ni}_{2.445}\left(0_{2}^{+}\right)$ & 0.228 \\
& $\left(1 f_{5 / 2}\right)$ & & -0.129 \\
${ }^{65} \mathrm{Ni}_{1.27}(5 / 2-)$ & $\left(1 g_{9 / 2}\right)$ & ${ }^{66} \mathrm{Ni}_{2.916}\left(2_{2}^{+}\right)$ & 0.483 \\
& $\left(2 p_{1 / 2}\right)$ & & -0.077 \\
${ }^{65} \mathrm{Ni}_{1.41}(1 / 2-)$ & $\left(2 p_{1 / 2}\right)$ & ${ }^{66} \mathrm{Ni}_{g . s}\left(0_{1}^{+}\right)$ & 0.029 \\
${ }^{65} \mathrm{Ni}_{1.41}\left(1 / 2_{2}^{-}\right)$ & $\left(2 p_{1 / 2}\right)$ & ${ }^{66} \mathrm{Ni}_{2.445}\left(0_{2}^{+}\right)$ & 0.578 \\
\hline \hline
\end{tabular}

Regarding the results of the independent coordinate and sequential models described in Secs. III B, III C, and III D one can conclude that for the ground state of ${ }^{66} \mathrm{Ni}$, using the shell-model spectroscopic amplitudes, the two resulting calculations are very similar (Fig. 4). On the other hand, using the microscopic IBM-2 spectroscopic amplitudes, the direct transfer reaction mechanism is dominant and in good agreement with the experimental data, while the sequential model underestimates the data (Fig. 7).

Concerning the first excited state of ${ }^{66} \mathrm{Ni}$, the results of the sequential transfer calculations with shell-model amplitudes are closer to the experimental data with respect to the independent coordinate angular distribution (Fig. 4), but not satisfactory. Instead, the sequential process obtained within the IBFM-2 (Fig. 7) describes well the experimental data, while the direct process angular distribution is more than one order of magnitude lower than the data.

The observation that for the transition to the $0^{+}$ground state there is a dominance of the direct mechanism while for the $2^{+}$state the sequential contribution is more important allows us to conclude that the pairing correlations among the two transferred neutrons are relevant mainly for the ground state. This state has a weak collectivity because the ${ }^{66} \mathrm{Ni}$ is an even-even spherical nucleus. Conversely, the $2^{+}$ state is a collective state, so that the long-range correlations between nucleons are dominant over the short-range pairing correlations of the two neutrons. The results obtained here for this excited state are quite different to the conclusions obtained recently for the transfer to the excited states of ${ }^{14,15} \mathrm{C}[3,36]$ and ${ }^{18} \mathrm{O}$ [14]. In such light systems, the collectivity of $2^{+}$ first excited states is sensibly smaller than for ${ }^{66} \mathrm{Ni}\left(2^{+}\right)$. As a result long-range correlations, associated with the quadrupole deformation, are weaker for the explored carbon and oxygen isotopes, making the effects of short-range pairing correlations particularly evident there. Table $\mathrm{X}$ shows the reduced electric quadrupole transition probability $[\mathrm{B}(\mathrm{E} 2)]$ between the ground state and the first $2^{+}$excited state for which the $\left({ }^{18} \mathrm{O},{ }^{16} \mathrm{O}\right)$ reaction was studied [42].
TABLE X. Reduced electric quadrupole transition probabilities for some selected nuclei [55].

\begin{tabular}{lc}
\hline \hline Nucleus & $\mathrm{B}(\mathrm{E} 2) ; 0^{+} \rightarrow 2^{+}\left(\mathrm{e}^{2} \mathrm{~b}^{2}\right)$ \\
\hline${ }^{14} \mathrm{C}$ & 0.0018 \\
${ }^{18} \mathrm{O}$ & 0.0045 \\
${ }^{28} \mathrm{Mg}$ & 0.035 \\
${ }^{66} \mathrm{Ni}$ & 0.060 \\
${ }^{76} \mathrm{Ge}$ & 0.270 \\
\hline \hline
\end{tabular}

From Table $\mathrm{X}$ one can see that the $\mathrm{B}(\mathrm{E} 2)$ are smaller for the ${ }^{14} \mathrm{C}$ and ${ }^{18} \mathrm{O}$. These are the two nuclei for which the transfer to the $2^{+}$state is dominated by the one-step two-neutron transfer mechanism. For ${ }^{28} \mathrm{Mg}$ M. Bernas et al. [21], despite their limited theoretical analysis, showed that the one-step mechanism described the transfer to the ground state, while it gave one order of magnitude lower that the experimental data for the transfer to the $2^{+}$state. Also, Lemaire and Low [8] obtained similar results, as we obtained here for ${ }^{66} \mathrm{Ni}$, for the two-neutron transfer to the ${ }^{76} \mathrm{Ge}$ nucleus. From Table $\mathrm{X}$, one realizes that the $\mathrm{B}(\mathrm{E} 2)$ value for ${ }^{28} \mathrm{Mg}$ and ${ }^{76} \mathrm{Ge}$ is also higher than the ones for the carbon and oxygen isotopes. This indicates that when the collectivity of the state increases the effect of pairing correlations in the two-neutron transfer reaction might be not evident.

\section{SUMMARY AND CONCLUSIONS}

In the present work high-quality angular distributions for the ${ }^{64} \mathrm{Ni}\left({ }^{18} \mathrm{O},{ }^{16} \mathrm{O}\right){ }^{66} \mathrm{Ni}$ transfer reactions were measured. The data were analyzed using three theoretical models: the extreme cluster model, independent coordinate, and twostep DWBA model. The spectroscopic amplitudes needed in microscopic calculations were calculated using two structure models: the shell model and the interacting boson model. The nickel isotopes are in fact in the upper limit of confident applicability of the shell model and the IBM is about its lower limit.

It was shown that the extreme cluster model is not a good approximation to describe the experimental two-neutron angular distributions for the present reactions, because this model overestimates the cross sections in the whole angular range where the angular distribution was measured.

The two-step DWBA and the independent coordinate models were used to perform microscopic calculations. The former was used to describe the sequential transfer process, while the independent coordinate models was used to describe the direct transfer reactions.

For the two-neutron transfer reactions to the ground state of the residual nucleus ${ }^{66} \mathrm{Ni}$, we have evidence of the short-range pairing correlations, using both nuclear structure models. This conclusion is more evident when we used the microscopic IBM-2 and the IBFM-2. In the two-neutron transfer to the first excited state of ${ }^{66} \mathrm{Ni}$, where the collectivity is known to be important, it was verified the predominance of the two-step reaction mechanism. This confirmed the long-range predominance in the wave function of this state. This conclusion was again 
independent on the nuclear structure model used for the calculations of the spectroscopic amplitudes of the target overlaps.

It would be interesting to measure and perform similar calculations for other systems with different masses and B(E2) values to verify our conclusions.

\section{ACKNOWLEDGMENTS}

The Brazilian authors acknowlede partial financial support from CNPq, FAPERJ, and CAPES and from INCT-FNA (Instituto Nacional de Ciência e Tecnologia- Física Nuclear e Aplicações), research project 464898/2014-5.
[1] G. R. Satchler, Direct Nuclear Reactions (Oxford University Press, Oxford, 1983).

[2] R. A. Broglia and A. Winther, Heavy Ion Reactions (Westview Press, Boulder, 2004).

[3] M. Cavallaro, F. Cappuzzello, M. Bondì, D. Carbone, V. N. Garcia, A. Gargano, S. M. Lenzi, J. Lubian, C. Agodi, F. Azaiez et al., Phys. Rev. C 88, 054601 (2013).

[4] M. Cavallaro, C. Agodi, M. Assié, F. Azaiez, F. Cappuzzello, D. Carbone, N. de Séréville, A. Foti, L. Pandola, J. A. Scarpaci et al., Phys. Rev. C 93, 064323 (2016).

[5] D. Carbone, F. Cappuzzello, M. Cavallaro, A. Cunsolo, A. Foti, S. Tudisco, M. Bondi, G. Santagati, G. Taranto, R. Chen et al., J. Phys.: Conf. Ser. 312, 082016 (2011).

[6] T. Takemasa and H. Yoshida, Nucl. Phys. A 304, 229 (1978).

[7] P. D. Bond, H. J. Korner, M. C. Lemaire, D. J. Pisano, and C. E. Thorn, Phys. Rev. C 16, 177 (1977).

[8] M. C. Lemaire and K. S. Low, Phys. Rev. C 16, 183 (1977).

[9] G. Potel, A. Idini, F. Barranco, E. Vigezzi, and R. A. Broglia, Rep. Prog. Phys. 76, 106301 (2013).

[10] I. Inci and A. Vitturi, J. Phys.: Conf. Ser. 321, 012004 (2011).

[11] W. von Oertzen and A. Vitturi, Rep. Prog. Phys. 64, 1247 (2001).

[12] T. Kammuri, Nucl. Phys. A 259, 343 (1976).

[13] F. Cappuzzello, D. Carbone, M. Cavallaro, M. Bondì, C. Agodi, F. Azaiez, A. Bonaccorso, A. Cunsolo, L. Fortunato, A. Foti et al., Nat. Commun. 6, 6743 (2015).

[14] F. Cappuzzello, M. Cavallaro, C. Agodi, M. Bondì, D. Carbone, A. Cunsolo, and A. Foti, Eur. Phys. J. A 51, 145 (2015).

[15] F. Cappuzzello, C. Agodi, M. Bondì, D. Carbone, M. Cavallaro, and A. Foti, J. Phys.: Conf. Ser. 630, 012018 (2015).

[16] M. Ermamatov, F. Cappuzzello, J. Lubian, M. Cubero, C. Agodi, D. Carbone, M. Cavallaro, J. L. Ferreira, A. Foti, V. N. Garcia et al., Phys. Rev. C 94, 024610 (2016).

[17] J. F. P. Huiza, E. Crema, A. Barioni, D. S. Monteiro, J. M. B. Shorto, R. F. Simoes, and P. R. S. Gomes, Phys. Rev. C 82, 054603 (2010).

[18] E. Crema, D. R. Otomar, R. F. Simoes, A. Barioni, D. S. Monteiro, L. K. Ono, J. M. B. Shorto, J. Lubian, and P. R. S. Gomes, Phys. Rev. C 84, 024601 (2011).

[19] S. Kahana and A. J. Baltz, Advances in Nuclear Physics, Vol. 9 (Springer, Berlin, 1977).

[20] E. H. Auerbach, A. J. Baltz, P. D. Bond, C. Chasman, J. D. Garrett, K. W. Jones, S. Kahana, M. J. LeVine, M. Schneider, A. Z. Schwarzschild et al., Phys. Rev. Lett 30, 1078 (1973).

[21] M. Bernas, M. Roy-Stephan, F. Pougheon, M. Langevin, G. Rotbard, P. Roussel, J. P. LeFèvre, M. C. Lemaire, K. S. Low, and B. H. Wildenthal, Phys. Rev. C 19, 2246 (1979).

[22] A. Arima and F. Iachello, Phys. Rev. Lett. 35, 1069 (1975).

[23] F. Iachello and P. V. Sacker, The interacting Boson-Fermion Model (Cambridge University, Cambridge, 1991).

[24] M. Cavallaro, F. Cappuzzello, M. Bondì, D. Carbone, V. N. Garcia, A. Gargano, S. M. Lenzi, J. Lubian, C. Agodi, F. Azaiez et al., EPJ Web Conf. 66, 03017 (2014).
[25] F. Cappuzzello, C. Agodi, D. Carbone, and M. Cavallaro, Eur. Phys. J. A 52, 167 (2016).

[26] L. Coraggio, A. Covello, A. Gargano, and N. Itaco, Phys. Rev. C 89, 024319 (2014).

[27] A. Cunsolo, F. Cappuzzello, M. Cavallaro, A. Foti, A. Khouaja, S. E. A. Orrigo, J. S. Winfield, L. Gasparini, G. Longo, T. Borello-Lewin et al., Eur. Phys. J. Special Topics 150, 343 (2007).

[28] M. Cavallaro, F. Cappuzzello, D. Carbone, A. Cunsolo, A. Foti, A. Khouaja, M. R. D. Rodrigues, J. S. Winfield, and M. Bondì, Eur. Phys. J. A 48, 59 (2012).

[29] F. Cappuzzello, M. Cavallaro, A. Cunsolo, A. Foti, D. Carbone, S. Orrigo, and M. Rodrigues, Nucl. Inst. Meth. Phys. Res. Sect. A 621, 419 (2010).

[30] F. Cappuzzello, D. Carbone, and M. Cavallaro, Nucl. Inst. Meth. Phys. Res. Sect. A 638, 74 (2011).

[31] F. Cappuzzello, C. Agodi, M. Bondì, D. Carbone, M. Cavallaro, A. Cunsolo, M. D. Napoli, A. Foti, D. Nicolosi, S. Tropea et al., Nucl. Instrum. Methods Phys. Res. Sect. A 763, 314 (2014).

[32] D. Carbone, Eur. Phys. J. Plus 130, 143 (2015).

[33] W. Darcey, R. Chapman, and S. Hinds, Nucl. Phys. A 170, 253 (1971).

[34] O. Scholten and Z. R. Yu, Phys. Lett. B 161, 13 (1985).

[35] D. M. Brink, Semi-Classical Methods for Nucleus-Nucleus Scattering (Cambridge University Press, Cambridge, 1985).

[36] M. Cavallaro, M. Colonna, F. Cappuzzello, C. Agodi, M. Bondì, D. Carbone, A. Cunsolo, M. D. Napoli, and A. Foti, J. Phys: Conf. Ser. 515, 012003 (2014).

[37] I. J. Thompson, Comput. Phys. Rep. 7, 167 (1988).

[38] L. C. Chamon, D. Pereira, M. S. Hussein, M. A. Candido Ribeiro, and D. Galetti, Phys. Rev. Lett. 79, 5218 (1997).

[39] D. Pereira, J. Lubian, J. Oliveira, D. Sousa, and L. Chamon, Phys. Lett. B 670, 330 (2009).

[40] L. R. Gasques, L. C. Chamon, P. R. S. Gomes, and J. Lubian, Nucl. Phys. A 764, 135 (2006).

[41] D. Carbone, J. Ferreira, F. Cappuzzello, J. Lubian, C. Agodi, M. Cavallaro, A. Foti, A. Gargano, S. Lenzi, R. Linares et al., Phys. Rev. C 95, 034603 (2017).

[42] S. Raman, C. W. Nestor Jr., and P. Tikkanen, At. Data Nucl. Data Tables 78, 1 (2001).

[43] M. Moshinsky, Nucl. Phys. 13, 104 (1959).

[44] W. D. M. Rae, http://www.garsington.eclipse.co.uk.

[45] S. Bogner, T. T. S. Kuo, and L. Coraggio, Nucl. Phys. A 684, 432c (2001).

[46] S. Bogner, T. T. S. Kuo, L. Coraggio, A. Covello, and N. Itaco, Phys. Rev. C 65, 051301(R) (2002).

[47] A. P. Zuker, B. Buck, and J. B. McGrory, Phys. Rev. Lett 21, 39 (1968).

[48] J. Barea and F. Iachello, Phys. Rev. C 79, 044301 (2009).

[49] A. de Shalit and I. Talmi, Nuclear Shell Theory (Academic Press, New York, 1963). 
[50] A. Arima, T. Otsuka, F. Iachello, and I. Talmi, Phys. Lett. B 66 205 (1977)

[51] T. Otsuka, A. Arima, and F. Iachello, Nucl. Phys. A 309, 1 (1978).

[52] P. D. Duval and B. R. Barrett, Phys. Rev. C 24, 1272 (1981).
[53] S. Pittel, B. Barret, and P. Duval, Ann. Phys. 144, 168 (1982).

[54] E. Santopinto, R. M. Vsevolodovna, and H. Garcia-Tecocoatzi (unpublished).

[55] https://www.nndc.bnl.gov. 